\title{
INTERFEROMETER MEASUREMENTS OF WAVE LENGTHS IN THE VACUUM ARC SPECTRA OF TITA- NIUM AND OTHER ELEMENTS
}

\author{
By C. C. Kiess
}

\begin{abstract}
Wave-length determinations by means of the Fabry-Perot interferometer have been made for the vacuum are spectrum of titanium. More than 300 lines have been measured between $6743 \mathrm{~A}$ in the red and $2941 \mathrm{~A}$ in the ultra-violet. For many of the lines the accuracy of measurement exceeds 1 part in 6,000,000; for the majority it exceeds 1 part in 4,500,000. Using only the wave numbers of lines of wave lengths longer than $4700 \mathrm{~A}$, and constant wave-number differences as determined in various parts of the spectrum, a set of terms has been derived, which have been used to calculate the wave lengths of the blue and violet lines. The comparison of calculated with observed wave lengths indicates that no difference in scale exists between the red and violet regions. In addition to titanium some wave lengths were measured for iron, copper, calcium, barium, and other elements.
\end{abstract}

\section{CONTENTS}

II. Method of observation

III. Results for titanium

1. Description of Bureau of Standards wave lengths

2. Comparison with other observations

3. Computation of wave lengths from spectral terms _...

IV. Results for other elements...

1. Iron

2. Copper

3. $\mathrm{Na}, \mathrm{Al}, \mathrm{Ca}, \mathrm{V}, \mathrm{Cr}, \mathrm{Mn}, \mathrm{Ni}, \mathrm{Ba} \ldots$

V. Acknowledgments.... 90

\section{INTRODUCTION}

In the summer of 1923 the Allegheny Observatory and the spectroscopy section of the Bureau of Standards entered upon a cooperative program for the determination of standard solar wave lengths by interference methods. The results of that work, wave lengths of more than 700 Fraunhofer lines included between 3592 and 7148 A, have now been published. ${ }^{1}$ From the earlier results of the solar work it became apparent that a comparison of solar with laboratory wave lengths would have significance only if the latter were obtained from sources unaffected by conditions not present in the sun. It was,

1 Publ. Allegheny Observatory, 6, p. 105; 1926; pp. 125 and 141; 1927. 
therefore, decided to enlarge the scope of the program to include the determination of the vacuum are wave lengths of the more prominent elements appearing in the solar spectrum.

In the present paper are given the results which have been obtained for the element titanium from $6743 \mathrm{~A}$ in the red to $2941 \mathrm{~A}$ in the ultra-violet. Some wave lengths of other elements were also measured during the course of the work, and these are presented at this time because of their significance for the solar problem.

\section{METHOD OF OBSERVATION}

Light rays from the vacuum are and from the neon tube, which served as the source of standards, were delivered simultaneously to the Fabry-Perot interferometer. The vacuum are was identical with that designed and described by Curtis. ${ }^{2}$ The beam from the arc, perpendicular to the line joining the $\mathrm{Ne}$ tube and the slit of the spectrograph, was collimated with the beam from the Ne lamp by means of a thinly silvered diagonal mirror placed about $20 \mathrm{~cm}$ in front of the interferometer. The arrangement of the sources was in all respects similar to that used previously in the redetermination of secondary standards from the iron arc. ${ }^{3}$

The material used in the arc was powdered titanium carbide which was packed into a cored graphite rod that served as the lower and positive electrode. The upper, negative electrode was usually a copper rod, but for a few exposures, an upper graphite electrode was employed. The are operated at 240 volts, d. c. and 8 amperes, and was struck when the bell surrounding it was partially exhausted. When the chamber was reduced to about $10 \mathrm{~mm} \mathrm{Hg}$ pressure, as indicated by a vacuum gauge, the arc burned steadily for periods approximating an hour without renewal or readjustment of the electrodes. When exposures were in progress the electrodes were separated by $1 \mathrm{~cm}$, and the image projected onto the diaphragm of the interferometer was enlarged about 2.5 times.

Three series of observations were made in all. The first two were obtained with glass interferometer plates with $\mathrm{Ag}$ films cathodically deposited. For these two series a glass achromat was used to project the light from the interferometer onto the slit of the spectrograph. Invar etalons of $5,7.5,12,15,20$, and $25 \mathrm{~mm}$ thickness were used to separate the plates. In the first series of observations the small camera which takes a flat plate 6 by $20 \mathrm{~cm}$ was used. The region covered was from 5500 to $7500 \mathrm{~A}$. In the second and third series of observations the large camera was used. This takes a thin plate 6 by $40 \mathrm{~cm}$, which can be bent over a template to fit the

2 J. Opt. Soc. Am. and Rev. Sci. Inst., 8, p. 697; 1924.

3 B. S. Sci. Papers, No. 478, 19, p. 263; 1924. 
focal curve of the grating. The second set of observations extended from 3650 to $6550 \mathrm{~A}$, and the third from 2820 to $6550 \mathrm{~A}$. The interferometer plates used for the third set were of crystalline quartz and were thinly sputtered with $\mathrm{Pt}$ films, and for projecting the rings it was necessary to use the quartz-fluorite achromat. Etalons of 3, $5,6,7.5,8,12,15$, and $20 \mathrm{~mm}$ were used as separators for the quartz plates.

The spectrograph used for all the work was the one carrying the 7,500-lines per inch Anderson concave grating. It has been described elsewhere. ${ }^{4}$ The first series of observations was obtained on Eastman 33 plates sensitized with pinacyanol. For the second and third series either experimental plates prepared in the bureau's emulsion laboratory or Schleussner ultrarapid plates were used. Half of each long plate was panchromatized by bathing in a mixture of pantochrome and pinacyanol for recording the green, yellow, and red rays.

For the first series of plates the method of reduction employed in previous work was followed. ${ }^{5}$ For the second and third series, however, a slight modification of that described by Robertson ${ }^{6}$ or by Childs ${ }^{7}$ was adopted. A similar procedure was adopted for the reduction of some of the solar observations mentioned above. ${ }^{8}$

\section{RESULTS FOR TITANIUM}

\section{DESCRIPTIGN OF BUREAU OF STANDARDS WAVE LENGTHS}

Table 1 contains the wave lengths which have been measured in the vacuum arc spectrum of titanium in terms of the neon standards. The values of the Ne lines used for finding the interferometer thicknesses are those of Burns, Meggers, and Merrill. ${ }^{9}$ The justification for using these lines as standards instead of the primary standard, the red ray of cadmium, lies not only in the ease and economy with which they may be produced, but also in the fact that in the determination of secondary standards in spectra where exceedingly high orders of interference are not possible, they lead to results of the same precision as is obtained with the use of the Cd standard. This is demonstrated by the recent work of Babcock ${ }^{10}$ on the iron spectrum.

The wave lengths entered in the first column of Table 1 are the means of the individual results derived from all the plates. These values are for air at $15^{\circ} \mathrm{C}$. and $760 \mathrm{~mm} \mathrm{Hg}$ pressure. The results

\footnotetext{
${ }_{4}^{4}$ B. S. Sci. Papers, Nos. 312, 441, and 499.

5 B. S. Sci. Papers, Nos. 251, 274, 302, 327, 329, 441, 478, and 479.

${ }^{6}$ J. Opt. Soc. Am. and Rev. Sci. Inst., 9, p. 611; 1924.

7 J. Sci. Inst., 3, pp. 97 and 129; 1926.

8 Publ. Allegheny Observatory, 6, p. 126; 1927.

- B. S. Sci. Paper No. 329, 14, p. 765; 1918.

${ }^{10}$ Astrophys. J., 66, p. 256; 1927.
} 
from the $\mathrm{Ag}$ films required correction for dispersion of phase change which was determined by the method of short and long etalons as described by Meggers. ${ }^{11}$ The phase change curve is illustrated in Figure 1. No phase change correction was apparent for the results obtained with the $\mathrm{Pt}$ films in the region under investigation. However, when the means of the wave lengths obtained with the Pt films were compared with the means of all the observations, it was found that they were systematically higher by $0.001 \mathrm{~A}$ from 5150 to $4850 \mathrm{~A}$ by $0.002 \mathrm{~A}$ from 4850 to $4070 \mathrm{~A}$; and by $0.003 \mathrm{~A}$ from 4070 to $3650 \mathrm{~A}$. Accordingly, the wave lengths in Table 1 which were derived only from the Pt films have been corrected by the above amounts, the



$\lambda$ A. $U$.

FIG. 1.-Dispersion of phase change at reflection from silver films

correction $0.003 \mathrm{~A}$ being extrapolated to apply to all the lines shorter than $3650 \mathrm{~A}$. The justification for this procedure is given below in the discussion on the calculation of wave lengths from spectral terms.

In columns 2 and 3 of Table 1 are entered the number of observations from which the mean values of the wave lengths were derived, and letters indicating the probable errors of the determinations. The probable errors are expressed in parts per million; thus the letter A denotes a p. e. of 1 part in $6,000,000 ; B, 1$ part in $4,500,000$; C, 1 part in $3,000,000$; and $\mathrm{D}$, probable errors greater than 1 in $3,000,000$. The fourth column of the table contains the intensities and temperature classes of the lines as determined by King. ${ }^{12}$ Lines emitted

11 B. S. Sci. Papers No. 251, 12, p. 199; 1915.

12 Astrophys. J., 39, p. 139; 1914; 59, p. 155; 1924. 
by the ionized $\mathrm{Ti}$ atom are designated in this column by the symbol Ti II. The remaining three columns of the table contain data the significance of which is discussed below.

TABLE 1.-Vacuum arc wave lengths of titanium

\begin{tabular}{|c|c|c|c|c|c|c|}
\hline$\lambda$ I. A. & \begin{tabular}{|l} 
Num- \\
ber of \\
obser- \\
va- \\
tions
\end{tabular} & Weight & $\begin{array}{l}\text { Intensity and } \\
\text { temperature class }\end{array}$ & $\nu$ (Vac) & $\begin{array}{l}\text { Term combi- } \\
\text { nation }\end{array}$ & $(\mathrm{O}-\mathrm{C}) \lambda$ \\
\hline $\begin{array}{l}6743.124 \\
6556.066 \\
6554.226 \\
6546.276 \\
6366.354\end{array}$ & $\begin{array}{r}6 \\
17 \\
16 \\
12 \\
6\end{array}$ & $\begin{array}{l}\mathrm{A} \\
\mathrm{A} \\
\mathrm{A} \\
\mathrm{A} \\
\mathrm{C}\end{array}$ & $\begin{array}{l}10 \mathrm{III} A \\
25 \mathrm{III} \\
30 \mathrm{III} \\
20 \mathrm{III} \\
8 \mathrm{III}\end{array}$ & $\begin{array}{l}14825.836 \\
15248.847 \\
15253.126 \\
15271.650 \\
15703.246\end{array}$ & $\begin{array}{l}a \mathrm{D}^{\prime}-a \mathrm{D} \\
b^{3} \mathrm{~F}_{4}-d^{3} \mathrm{~F}^{\prime}{ }_{4} \\
b^{3} \mathrm{~F}_{3}-d^{3} \mathrm{~F}^{\prime}{ }^{\prime} \\
b^{3} \mathrm{~F}_{2}-d^{3} \mathrm{~F}^{\prime}{ }_{2} \\
b^{3} \mathrm{~F}_{4}-c^{3} \mathrm{D}_{3}\end{array}$ & $\begin{array}{r}000 \\
000 \\
000 \\
+004\end{array}$ \\
\hline $\begin{array}{l}6336.104 \\
6312.240 \\
6303.754 \\
6261.101 \\
6258.706\end{array}$ & $\begin{array}{r}2 \\
6 \\
6 \\
26 \\
23 \\
23\end{array}$ & $\begin{array}{l}\mathrm{D} \\
\mathrm{C} \\
\mathrm{C} \\
\mathrm{A} \\
\mathrm{A}\end{array}$ & $\begin{array}{l}8 \mathrm{III} \\
10 \mathrm{III} \\
10 \mathrm{III} \\
35 \mathrm{II} \\
50 \mathrm{II}\end{array}$ & $\begin{array}{l}15778.216 \\
15837.866 \\
15859.188 \\
15967.225 \\
15973.336\end{array}$ & $\begin{array}{l}b^{3} \mathrm{~F}_{3}-c^{3} \mathrm{D}_{2} \\
b^{3} \mathrm{~F}_{4}-b^{3} \mathrm{G}_{4} \\
b^{3} \mathrm{~F}_{3}-b^{3} \mathrm{G}_{3} \\
b^{3} \mathrm{~F}_{2}-b^{3} \mathrm{G}_{3} \\
b^{3} \mathrm{~F}_{4}-b^{3} \mathrm{G}_{5}\end{array}$ & $\begin{array}{r}000 \\
+003 \\
+010 \\
-001 \\
000\end{array}$ \\
\hline $\begin{array}{l}6258.103 \\
6126.217 \\
6091.175 \\
6085.228 \\
6064.631\end{array}$ & $\begin{array}{c}24 \\
16 \\
16 \\
16 \\
3\end{array}$ & $\begin{array}{l}\mathrm{A} \\
\mathrm{A} \\
\mathrm{B} \\
\mathrm{A} \\
\mathrm{B}\end{array}$ & $\begin{array}{l}40 \mathrm{II} \\
20 \mathrm{II} \\
20 \mathrm{III} \\
20 \mathrm{II} \\
9 \mathrm{IIA}\end{array}$ & $\begin{array}{l}15974.875 \\
16318.780 \\
16412.662 \\
16428.700 \\
16484.496\end{array}$ & $\begin{array}{c}b^{3} \mathrm{~F}_{3}-b^{3} \mathrm{G}_{4} \\
a^{3} \mathrm{P}_{2}-a^{3} \mathrm{~S}_{1} \\
b \mathrm{G}^{\prime}-b \mathrm{G} \\
a^{3} \mathrm{P}_{1}-a^{3} \mathrm{~S}_{1} \\
a^{3} \mathrm{P}_{0}-a^{3} \mathrm{~S}_{1}\end{array}$ & 000 \\
\hline $\begin{array}{l}5999.668 \\
5978.543 \\
5965.828 \\
5953.162 \\
5941.755\end{array}$ & $\begin{array}{r}1 \\
20 \\
24 \\
22 \\
7\end{array}$ & $\begin{array}{l}\mathrm{D} \\
\mathrm{A} \\
\mathrm{A} \\
\mathrm{A} \\
\mathrm{B}\end{array}$ & $\begin{array}{l}8 \mathrm{III} \\
25 \mathrm{II} \\
30 \mathrm{II} \\
30 \mathrm{II} \\
12 \mathrm{II} A\end{array}$ & $\begin{array}{l}16662.984 \\
16721.863 \\
16757.501 \\
16793.155 \\
16825.395\end{array}$ & $\begin{array}{c}a^{3} \mathrm{H}_{4}-b G \\
a^{3} G_{3}^{\prime}-a^{3} H^{\prime} \\
a^{3} G^{\prime}{ }^{3}-a^{3} \mathrm{H}^{\prime}{ }_{3} \\
a^{3} \mathrm{G}^{4}-a^{3} \mathrm{H}^{\prime} 6 \\
a^{3} \mathrm{P}_{1}-b^{3} \mathrm{D}_{1}\end{array}$ & $\begin{array}{r}000 \\
000 \\
000 \\
+005\end{array}$ \\
\hline $\begin{array}{l}5937.806 \\
5922.112 \\
5918.548 \\
5903.317 \\
5899.295\end{array}$ & $\begin{array}{r}1 \\
7 \\
2 \\
1 \\
22\end{array}$ & $\begin{array}{l}\mathrm{D} \\
\mathrm{C} \\
\mathrm{D} \\
\mathrm{D} \\
\mathrm{A}\end{array}$ & $\begin{array}{l}6 \mathrm{II} A \\
18 \mathrm{II} \\
10 \mathrm{II} \\
5 \mathrm{III} A \\
25 \mathrm{II}\end{array}$ & $\begin{array}{l}16836.584 \\
16881.201 \\
16891.367 \\
16934.947 \\
16946.493\end{array}$ & $\begin{array}{l}a^{3} \mathrm{P}_{2}-b^{3} \mathrm{D}_{2} \\
a^{3} \mathrm{P}_{0}-b^{3} \mathrm{D}_{1} \\
a^{3} \mathrm{P}_{2}-a^{3} \mathrm{P}^{\prime}{ }_{2} \\
a^{3} \mathrm{P}_{2}-a^{3} \mathrm{P}^{\prime}{ }_{1} \\
a^{3} \mathrm{P}_{1}-b^{3} \mathrm{D}_{2}\end{array}$ & $\begin{array}{l}-002 \\
+004\end{array}$ \\
\hline $\begin{array}{l}5866.453 \\
5804.265 \\
5785.979 \\
5774.037 \\
5766.330\end{array}$ & $\begin{array}{r}31 \\
8 \\
3 \\
6 \\
3\end{array}$ & $\begin{array}{l}\text { A } \\
\text { C } \\
\text { B } \\
\text { C } \\
\text { D }\end{array}$ & $\begin{array}{l}35 \mathrm{II} \\
5 n \mathrm{IV} \\
5 n \mathrm{IV} \\
5 n \mathrm{IV} \\
4 n \mathrm{IV}\end{array}$ & $\begin{array}{l}17041.364 \\
17223.947 \\
17278.379 \\
17314.116 \\
17337.257\end{array}$ & $\begin{array}{l}a^{3} \mathrm{P}_{2}-b^{3} \mathrm{D}_{3} \\
b^{5} \mathrm{G}_{6}-c^{5} \mathrm{H}_{7} \\
b^{5} \mathrm{G}_{5}-c^{5} \mathrm{H}_{6} \\
b^{5} \mathrm{G}_{4}-c^{5} \mathrm{H}_{5} \\
b^{5} \mathrm{G}_{3}-c^{5} \mathrm{H}_{4}\end{array}$ & +004 \\
\hline $\begin{array}{l}5739.464 \\
5715.123 \\
5689.465 \\
5575.413 \\
5662.154\end{array}$ & $\begin{array}{r}5 \\
3 \\
7 \\
10 \\
10\end{array}$ & $\begin{array}{l}\mathrm{B} \\
\mathrm{D} \\
\mathrm{B} \\
\mathrm{B} \\
\mathrm{A}\end{array}$ & $\begin{array}{l}9 \mathrm{III} \\
9 \mathrm{III} \\
10 \mathrm{III} \\
9 \mathrm{III} A \\
12 \mathrm{III}\end{array}$ & $\begin{array}{l}17418.410 \\
17492.594 \\
17571.481 \\
17614.987 \\
17656.237\end{array}$ & $\begin{array}{l}a^{3} \mathrm{H}_{5}-b^{3} \mathrm{H}^{\prime}{ }^{\prime} \\
a^{3} \mathrm{H}_{6}-b^{3} \mathrm{H}_{6}^{\prime} \\
a^{5} \mathrm{D}_{2}-b^{5} \mathrm{~F}_{3} \\
a^{5} \mathrm{D}_{3}-b^{5} \mathrm{~F}_{4} \\
a^{5} \mathrm{D}_{4}-b^{5} \mathrm{~F}_{5}\end{array}$ & $\begin{array}{l}000 \\
000\end{array}$ \\
\hline $\begin{array}{l}5648.570 \\
5644.137 \\
5565.476 \\
5512.529 \\
5503.897\end{array}$ & $\begin{array}{r}4 \\
13 \\
11 \\
17 \\
8\end{array}$ & $\begin{array}{l}\text { D } \\
\text { A } \\
\text { A } \\
\text { A } \\
\text { B }\end{array}$ & $\begin{array}{l}5 \mathrm{IV} \\
18 \mathrm{III} \\
9 \mathrm{III} \\
25 \mathrm{II} \\
8 \mathrm{III}\end{array}$ & $\begin{array}{l}17698.695 \\
17712.595 \\
17962.941 \\
18135.471 \\
18163.912\end{array}$ & $\begin{array}{c}a^{3} \mathrm{D}_{3}-c^{3} \mathrm{~F}_{4} \\
b \mathrm{G}^{\prime}-c \mathrm{G} \\
a^{3} \mathrm{H}_{4}-c \mathrm{G} \\
b^{3} \mathrm{~F}_{4}-e^{3} \mathrm{D}_{3} \\
a \mathrm{H}-d \mathrm{G}\end{array}$ & +003 \\
\hline $\begin{array}{l}5490.151 \\
5488.210 \\
5481.426 \\
5477.695 \\
5429.139\end{array}$ & $\begin{array}{r}10 \\
2 \\
3 \\
11 \\
5\end{array}$ & $\begin{array}{l}\mathrm{B} \\
\mathrm{D} \\
\mathrm{D} \\
\mathrm{B} \\
\mathrm{D}\end{array}$ & $\begin{array}{l}12 \text { II } \\
5 \text { III } \\
6 \text { III } \\
8 \text { III } \\
6 \text { III }\end{array}$ & $\begin{array}{l}18209.391 \\
18215.829 \\
18238.374 \\
18250.796 \\
18414.022\end{array}$ & $\begin{array}{c}b^{3} \mathrm{~F}_{4}-b^{5} \mathrm{D}_{3} \\
a^{3} \mathrm{~F}_{4}^{\prime}-2-c^{3} \mathrm{~F}_{2} \\
a^{3} \mathrm{~F}^{\prime} c^{3} \mathrm{~F}_{3} \\
a^{3} \mathrm{~F}^{\prime}{ }_{4}^{4}-c^{3} \mathrm{~F}_{4} \\
c^{3} \mathrm{P}_{2}-d^{3} \mathrm{P}^{\prime}{ }_{2}\end{array}$ & $\begin{array}{l}000 \\
000 \\
000\end{array}$ \\
\hline $\begin{array}{l}5409.609 \\
5397.093 \\
5389.996 \\
5369.635 \\
5298.429\end{array}$ & $\begin{array}{l}8 \\
5 \\
2 \\
3 \\
2\end{array}$ & $\begin{array}{l}C \\
D \\
D \\
D \\
D\end{array}$ & $\begin{array}{l}6 \mathrm{II} \\
4 \mathrm{III} \\
3 \mathrm{III} \\
4 \mathrm{III} \\
4 \mathrm{III}\end{array}$ & $\begin{array}{l}18480.500 \\
18523.359 \\
18547.745 \\
18618.077 \\
18868.284\end{array}$ & $\begin{array}{c}a^{3} \mathrm{G}_{5}^{\prime}-e^{3} \mathrm{~F}^{\prime}{ }_{4} \\
a^{3} \mathrm{G}_{4}^{\prime}-e^{3} \mathrm{~F}^{\prime}{ }_{3} \\
a^{3} \mathrm{G}_{3}^{\prime}-e^{3} \mathrm{~F}_{2}^{\prime} \\
b \mathrm{D}^{\prime}-c \mathrm{P}^{\prime}\end{array}$ & $\begin{array}{r}-001 \\
000 \\
000\end{array}$ \\
\hline $\begin{array}{l}\text { 5297. } 236 \\
5295.781 \\
5283.441 \\
5265.967 \\
5224.928\end{array}$ & $\begin{array}{l}3 \\
2 \\
4 \\
6 \\
3\end{array}$ & $\begin{array}{l}D \\
D \\
D \\
C \\
D\end{array}$ & $\begin{array}{l}6 \mathrm{III} \\
4 \mathrm{III} \\
8 \mathrm{III} \\
10 \mathrm{III} \\
8 \mathrm{III}\end{array}$ & $\begin{array}{l}18872.532 \\
18877.720 \\
18921.809 \\
18984.597 \\
19133.706\end{array}$ & $\begin{array}{r}a^{3} \mathrm{G}^{\prime}{ }_{3}-f^{3} \mathrm{~F}^{\prime}{ }_{2} \\
a^{3} \mathrm{P}_{2}-c^{3} \mathrm{D}_{3} \\
a^{3} \mathrm{G}_{4}^{\prime}-\mathrm{F}^{\prime} \mathrm{F}_{3} \\
a^{3} \mathrm{G}^{\prime}-f^{3} \mathrm{~F}_{4}{ }_{4}^{5} \mathrm{a}^{5} \mathrm{~F}^{\prime}-b^{5} \mathrm{~F}_{4}\end{array}$ & $\begin{array}{r}000 \\
+002 \\
000 \\
+602\end{array}$ \\
\hline
\end{tabular}

$2284^{\circ}-28-6$ 
TABLE 1.-Vacuum arc wave lengths of titanium-Continued

\begin{tabular}{|c|c|c|c|c|c|c|}
\hline$\lambda$ I. A. & $\begin{array}{l}\text { Num- } \\
\text { ber of } \\
\text { obser- } \\
\text { va- } \\
\text { tions }\end{array}$ & Weight & $\begin{array}{l}\text { Intensity and } \\
\text { temperature class }\end{array}$ & $\nu(\mathrm{Vac})$ & $\begin{array}{l}\text { Term combi- } \\
\text { nation }\end{array}$ & $(\mathrm{O}-\mathrm{C}) \lambda$ \\
\hline $\begin{array}{l}5224.301 \\
5210.386 \\
5192.971 \\
5173.742 \\
5147.483\end{array}$ & $\begin{array}{r}3 \\
29 \\
26 \\
27 \\
12\end{array}$ & $\begin{array}{l}\mathrm{D} \\
\mathrm{A} \\
\mathrm{A} \\
\mathrm{A} \\
\mathrm{A}\end{array}$ & $\begin{array}{l}15 \mathrm{III} \\
40 \mathrm{I} \\
35 \mathrm{I} \\
30 \mathrm{I} \\
10 \mathrm{I} A\end{array}$ & $\begin{array}{l}19136.006 \\
19187.107 \\
19251.453 \\
19323.003 \\
19421.575\end{array}$ & $\begin{array}{c}a^{3} \mathrm{~F}^{\prime}{ }_{5}-b^{5} \mathrm{~F}_{5} \\
a^{3} \mathrm{~F}_{4}-a^{3} \mathrm{~F}^{\prime}{ }_{4} \\
a^{3} \mathrm{~F}_{3}-a^{3} \mathrm{~F}^{\prime}{ }_{3} \\
a^{3} \mathrm{~F}_{2}-a^{3} \mathrm{~F}^{\prime}{ }^{2} \\
a^{3} \mathrm{~F}_{2}-a^{3} \mathrm{~F}^{\prime}{ }_{3}\end{array}$ & $\begin{array}{r}000 \\
-001 \\
000 \\
+001\end{array}$ \\
\hline $\begin{array}{l}5145.465 \\
5120.420 \\
5113.448 \\
5087.055 \\
5064.654\end{array}$ & $\begin{array}{r}13 \\
10 \\
8 \\
3 \\
26\end{array}$ & $\begin{array}{l}\mathrm{A} \\
\mathrm{A} \\
\mathrm{C} \\
\mathrm{D} \\
\mathrm{A}\end{array}$ & $\begin{array}{l}12 \text { II } \\
12 \text { III } \\
10 \text { III } \\
8 \text { III } \\
25 \text { I }\end{array}$ & $\begin{array}{l}19429.194 \\
19524.222 \\
19550.843 \\
19652.277 \\
19739.198\end{array}$ & $\begin{array}{l}b^{3} F_{4}-f^{3} D_{3} \\
b^{3} F_{3}-f^{3} D_{2} \\
b^{3} F_{2}-f^{3} D_{1} \\
a^{3} F_{4}-a^{3} D_{3}\end{array}$ & $\begin{array}{l}000 \\
000 \\
000 \\
000\end{array}$ \\
\hline $\begin{array}{l}5062.112 \\
5052.879 \\
5039.959 \\
5038.400 \\
5036.468\end{array}$ & $\begin{array}{r}2 \\
2 \\
22 \\
15 \\
10\end{array}$ & $\begin{array}{l}\text { D } \\
\text { D } \\
\text { A } \\
\text { A } \\
\text { B }\end{array}$ & $\begin{array}{l}7 \text { III } \\
8 \text { IV } \\
22 \text { I } \\
25 \text { II } \\
25 \text { II }\end{array}$ & $\begin{array}{l}\text { 19749. } 111 \\
19785.196 \\
19835.917 \\
19842.053 \\
19849.667\end{array}$ & $\begin{array}{c}a^{3} \mathrm{D}^{\prime}{ }_{2}-d^{3} \mathrm{P}^{\prime}{ }_{1} \\
a^{3} \mathrm{D}^{\prime}{ }_{3}-d^{3} \mathrm{P}^{\prime}{ }_{2} \\
a^{3} \mathrm{~F}_{3}-a^{3} \mathrm{D}_{2} \\
b^{3} \mathrm{~F}_{2}-d^{3} \mathrm{G}_{3} \\
b^{3} \mathrm{~F}_{3}-d^{3} \mathrm{G}_{4}\end{array}$ & $\begin{array}{r}000 \\
000 \\
000 \\
-001 \\
000\end{array}$ \\
\hline $\begin{array}{l}5035.908 \\
5025.570 \\
5024.842 \\
5022.871 \\
5020.028\end{array}$ & $\begin{array}{r}11 \\
9 \\
12 \\
22 \\
22\end{array}$ & $\begin{array}{l}\mathrm{B} \\
\mathrm{C} \\
\mathrm{C} \\
\mathrm{A} \\
\mathrm{A}\end{array}$ & $\begin{array}{l}25 \text { II } \\
18 \text { III } \\
20 \text { II } \\
25 \\
25 \text { II }\end{array}$ & $\begin{array}{l}\text { 19851. } 873 \\
19892.711 \\
19895.592 \\
19903.397 \\
19914.670\end{array}$ & $\begin{array}{l}b^{3} \mathrm{~F}_{4}-d^{3} \mathrm{G}_{5} \\
a^{5} \mathrm{G}_{6}-b^{5} \mathrm{~F}_{5} \\
a^{5} \mathrm{~F}_{2}-b^{5} \mathrm{G}_{2} \\
a^{5} \mathrm{~F}_{3}-b^{5} \mathrm{G}_{3} \\
a^{5} \mathrm{~F}_{4}-b^{5} \mathrm{G}_{4}\end{array}$ & +001 \\
\hline $\begin{array}{l}5016.162 \\
5014.277 \\
5014.185 \\
5009.652 \\
5007.209\end{array}$ & $\begin{array}{r}19 \\
28 \\
3 \\
1 \\
28\end{array}$ & $\begin{array}{l}\mathrm{A} \\
\mathrm{A} \\
\mathrm{D} \\
\mathrm{D} \\
\mathrm{A}\end{array}$ & $\begin{array}{l}20 \mathrm{II} \\
25 \mathrm{I} \\
25 \mathrm{I} \\
7 \mathrm{IA} \\
40 \mathrm{II}\end{array}$ & $\begin{array}{l}19930.026 \\
19937.512 \\
19937.878 \\
19955.917 \\
19965.651\end{array}$ & $\begin{array}{l}a^{5} \mathrm{~F}_{5}-b^{5} \mathrm{G}_{5} \\
a^{5} \mathrm{~F}_{1}-b^{5} \mathrm{G}_{2} \\
a^{3} \mathrm{~F}_{2}-a^{3} \mathrm{D}_{1} \\
a^{3} \mathrm{~F}_{3}-a^{3} \mathrm{D}_{3} \\
a^{5} \mathrm{~F}_{2}-b^{5} \mathrm{G}_{3}\end{array}$ & $\begin{array}{r}000 \\
+006\end{array}$ \\
\hline $\begin{array}{l}4999.504 \\
4997.099 \\
4991.067 \\
4981.732 \\
4978.191\end{array}$ & $\begin{array}{r}30 \\
2 \\
30 \\
30 \\
8\end{array}$ & $\begin{array}{l}\mathrm{A} \\
\mathrm{D} \\
\mathrm{A} \\
\mathrm{A} \\
\mathrm{C}\end{array}$ & $\begin{array}{l}45 \mathrm{II} \\
8 \mathrm{IA} \\
50 \mathrm{II} \\
60 \mathrm{II} \\
10 \mathrm{III}\end{array}$ & $\begin{array}{l}19996.425 \\
20006.046 \\
20030.226 \\
20067.757 \\
20082.031\end{array}$ & $\begin{array}{l}a^{5} \mathbf{F}_{3}-b^{5} \mathbf{G}_{4} \\
a^{3} \mathbf{F}_{2}-a^{3} D_{2} \\
a^{5} \mathbf{F}_{4}-b^{5} \mathbf{G}_{5} \\
a^{5} \mathbf{F}_{5}-b^{5} \mathbf{G}_{6} \\
a^{5} \mathbf{G}_{2}-b^{5} \mathbf{F}_{1}\end{array}$ & +001 \\
\hline $\begin{array}{l}4975.344 \\
4938.283 \\
4928.342 \\
4921.768 \\
4919.867\end{array}$ & $\begin{array}{r}10 \\
9 \\
13 \\
13 \\
11\end{array}$ & $\begin{array}{l}\mathrm{C} \\
\mathrm{D} \\
\mathrm{A} \\
\mathrm{B} \\
\mathrm{B}\end{array}$ & $\begin{array}{l}10 \mathrm{III} \\
8 \mathrm{IV} \\
12 \mathrm{III} \\
12 \mathrm{III} \\
10 \mathrm{III}\end{array}$ & $\begin{array}{l}20093.523 \\
20244.321 \\
20285.152 \\
20312.248 \\
20320.098\end{array}$ & $\begin{array}{c}b \mathrm{D}^{\prime}-d \mathrm{~F}^{\prime} \\
a \mathrm{H}-f \mathrm{G} \\
a^{3} \mathrm{D}^{\prime}{ }_{1}-g^{3} \mathrm{~F}^{\prime}{ }_{2} \\
a^{3} \mathrm{D}^{\prime}{ }_{3}-g^{3} \mathrm{~F}^{\prime}{ }_{4} \\
a^{3} \mathrm{D}_{2}^{\prime}{ }_{2}-g^{3} \mathrm{~F}^{\prime}{ }_{3}\end{array}$ & $\begin{array}{l}000 \\
000 \\
000\end{array}$ \\
\hline $\begin{array}{l}4913.616 \\
4899.910 \\
4885.082 \\
4870.129 \\
4868.264\end{array}$ & $\begin{array}{l}20 \\
24 \\
25 \\
15 \\
15\end{array}$ & $\begin{array}{l}\mathrm{A} \\
\mathrm{A} \\
\mathrm{A} \\
\mathrm{A} \\
\mathrm{A}\end{array}$ & $\begin{array}{l}20 \text { III } \\
20 \text { III } \\
20 \text { II } \\
20 \text { III } \\
18 \text { III }\end{array}$ & $\begin{array}{l}20345.946 \\
20402.859 \\
20464.788 \\
20527.621 \\
20535.483\end{array}$ & $\begin{array}{c}a^{3} \mathrm{G}^{\prime}{ }_{3}-b^{3} \mathrm{H}^{\prime}{ }_{4} \\
a^{3} \mathrm{G}^{\prime}{ }_{4}-b^{3} \mathrm{H}^{\prime}{ }_{5} \\
a^{3} \mathrm{G}_{5}^{\prime}-b^{3} \mathrm{H}^{\prime}{ }_{6} \\
a^{3} \mathrm{H}_{5}-a^{3} \mathrm{I}_{6} \\
a^{3} \mathrm{H}_{4}-a^{3} \mathrm{I}_{5}\end{array}$ & $\begin{array}{l}000 \\
000 \\
000\end{array}$ \\
\hline $\begin{array}{l}4856.012 \\
4840.874 \\
4820.410 \\
4805.416 \\
4799.797\end{array}$ & $\begin{array}{l}19 \\
25 \\
14 \\
13 \\
14\end{array}$ & $\begin{array}{l}\mathrm{A} \\
\mathrm{A} \\
\mathrm{A} \\
\mathrm{B} \\
\mathrm{B}\end{array}$ & $\begin{array}{l}20 \text { III } \\
25 \text { I } \\
20 \text { II } \\
12 \text { III } \\
12 \text { III }\end{array}$ & $\begin{array}{l}20587.298 \\
20651.676 \\
20739.345 \\
20804.051 \\
20828.412\end{array}$ & $\begin{array}{c}a^{3} \mathrm{H}_{6}-a^{3} \mathrm{I}_{7} \\
a \mathrm{D}^{\prime}-b \mathrm{D}^{\prime} \\
a \mathrm{G}^{\prime}-b \mathrm{~F}^{\prime} \\
c^{3} \mathrm{P}_{2}-i^{3} \mathrm{D}_{3} \\
b \mathrm{G}^{\prime}-c^{3} \mathrm{H}_{4}^{\prime}\end{array}$ & \\
\hline $\begin{array}{l}4792.482 \\
4778.259 \\
4759.272 \\
4758.120 \\
4742.791\end{array}$ & $\begin{array}{r}13 \\
9 \\
21 \\
18 \\
18\end{array}$ & $\begin{array}{l}\mathrm{A} \\
\mathrm{B} \\
\mathrm{A} \\
\mathrm{A} \\
\mathrm{A}\end{array}$ & $\begin{array}{l}10 \text { III } \\
10 \text { III } \\
25 \text { III } \\
25 \text { III } \\
20 \text { III }\end{array}$ & $\begin{array}{l}20860.203 \\
20922.292 \\
21005.760 \\
21010.849 \\
21078.756\end{array}$ & $\begin{array}{c}c^{3} \mathrm{P}_{1}-i^{3} \mathrm{D}_{2} \\
a^{3} \mathrm{H}_{4}-d \mathrm{G} \\
a^{3} \mathrm{H}_{6}-c^{3} \mathrm{H}^{\prime}{ }_{6} \\
a^{3} \mathrm{H}_{5}-c^{3} \mathrm{H}^{\prime}{ }_{5} \\
a^{3} \mathrm{H}_{4}-c^{3} \mathrm{H}_{4}^{\prime}\end{array}$ & \\
\hline $\begin{array}{l}\text { 4731. } 172 \\
4715.295\end{array}$ & $\begin{array}{l}7 \\
4\end{array}$ & $\begin{array}{l}\mathrm{A} \\
\mathrm{D}\end{array}$ & 9 III & $\begin{array}{l}21130.521 \\
21201.668\end{array}$ & $\begin{array}{c}a^{3} \mathrm{D}_{3}^{\prime}-h^{3} \mathrm{~F}^{\prime}{ }_{4} \\
a^{3} \mathrm{~F}_{4}-a^{3} \mathrm{G}_{4}\end{array}$ & -005 \\
\hline 4710. 186 & 15 & A & $18 \mathrm{III}$ & 21224.663 & $\left\{\begin{array}{c}a^{3} \mathrm{P}_{0}-e^{3} \mathrm{D}_{1} \\
a^{3} \mathrm{D}_{3}^{\prime}-h^{3} \mathrm{D}_{3}\end{array}\right.$ & \\
\hline 4698. 766 & 21 & A & $20 \mathrm{II}$ & 21276.247 & $a^{3} \mathrm{P}_{1}-e^{3} \mathrm{D}_{2}$ & 000 \\
\hline $\begin{array}{l}4691.336 \\
4681.908 \\
4675.118 \\
4667.585 \\
4656.468\end{array}$ & $\begin{array}{r}24 \\
30 \\
9 \\
30 \\
30\end{array}$ & $\begin{array}{l}\mathrm{A} \\
\mathrm{A} \\
\mathrm{C} \\
\mathrm{A} \\
\mathrm{A}\end{array}$ & $\begin{array}{l}20 \mathrm{II} \\
30 \mathrm{I} \\
10 \mathrm{III} \\
25 \mathrm{I} \\
25 \mathrm{I}\end{array}$ & $\begin{array}{l}21309.944 \\
21352.857 \\
21383.870 \\
21418.381 \\
21469.515\end{array}$ & $\begin{array}{l}a^{3} \mathrm{P}_{2}-e^{3} \mathrm{D}_{3} \\
a^{3} \mathrm{~F}_{4}-a^{3} \mathrm{G}_{5} \\
a^{3} \mathrm{P}_{2}-b^{5} \mathrm{D}_{3} \\
a^{3} \mathrm{~F}_{3}-a^{3} \mathrm{G}_{4} \\
a^{3} \mathrm{~F}_{2}-a^{3} \mathrm{G}_{3}\end{array}$ & $\begin{array}{l}-001 \\
+003 \\
+002 \\
+005\end{array}$ \\
\hline $\begin{array}{l}4645.193 \\
4629.336 \\
4623.098 \\
4617.269 \\
4599.226\end{array}$ & $\begin{array}{r}12 \\
24 \\
26 \\
29 \\
9\end{array}$ & $\begin{array}{l}\mathrm{C} \\
\mathrm{A} \\
\mathrm{A} \\
\mathrm{A} \\
\mathrm{A}\end{array}$ & $\begin{array}{l}12 \mathrm{III} \\
15 \mathrm{III} \\
25 \mathrm{III} \\
30 \mathrm{II} \\
5 \mathrm{IV}\end{array}$ & $\begin{array}{l}21521.626 \\
21595.343 \\
21624.478 \\
21651.780 \\
21736.720\end{array}$ & $\begin{array}{l}a^{5} \mathrm{P}_{1}-c^{5} \mathrm{D}_{0} \\
a^{5} \mathrm{P}_{1}-c^{5} \mathrm{D}_{2} \\
a^{5} \mathrm{P}_{2}-c^{5} \mathrm{D}_{3} \\
a^{5} \mathrm{P}_{3}-c^{5} \mathrm{D}_{4}\end{array}$ & \\
\hline
\end{tabular}


TABLE 1.-Vacuum arc wave lengths of itanium-Continued

\begin{tabular}{|c|c|c|c|c|c|c|}
\hline$\lambda I . A$. & $\begin{array}{c}\text { Num- } \\
\text { ber of } \\
\text { obser- } \\
\text { va- } \\
\text { tions }\end{array}$ & Weight & $\begin{array}{l}\text { Intensity and } \\
\text { temperature class }\end{array}$ & $\nu(\mathrm{Vac})$ & $\begin{array}{l}\text { Term combi- } \\
\text { nation }\end{array}$ & $(0-C) \lambda \mid$ \\
\hline $\begin{array}{l}4571.971 \\
4563.761 \\
4559.920 \\
4555.486 \\
4552.453\end{array}$ & $\begin{array}{r}23 \\
16 \\
7 \\
30 \\
30\end{array}$ & $\begin{array}{l}\text { A } \\
\text { A } \\
\text { B } \\
\text { A } \\
\text { A }\end{array}$ & $\begin{array}{l}15 \mathrm{VE}, \mathrm{Ti} \mathrm{II} \\
15 \mathrm{VE}, \mathrm{Ti} \\
6 \mathrm{III} \\
30 \mathrm{II} \\
35 \mathrm{II}\end{array}$ & $\begin{array}{l}21866.297 \\
21905.632 \\
21924.083 \\
21945.422 \\
21960.044\end{array}$ & $\begin{array}{l}a^{2} \mathrm{H}_{5}-a^{2} \mathrm{G}_{4} \\
a^{2} \mathrm{P}_{1}-a^{2} \mathrm{D}_{2} \\
b^{3} \mathrm{~F}_{4}-e^{3} \mathrm{~F}^{\prime}{ }_{4} \\
a^{5} \mathrm{~F}_{5}-b^{5} \mathrm{~F}^{\prime}{ }_{4} \\
a^{5} \mathrm{~F}_{4}-b^{5} \mathrm{~F}^{\prime}{ }_{3}\end{array}$ & -001 \\
\hline $\begin{array}{l}4549.622 \\
4548.764 \\
4544.688 \\
4533.238 \\
4527.305\end{array}$ & $\begin{array}{l}10 \\
29 \\
29 \\
21 \\
30\end{array}$ & $\begin{array}{l}\mathrm{C} \\
\mathrm{A} \\
\mathrm{A} \\
\mathrm{B} \\
\mathrm{A}\end{array}$ & $\begin{array}{l}25 \mathrm{VE} \text {, Ti II } \\
35 \mathrm{II} \\
30 \mathrm{II} \\
80 \mathrm{II} \\
35 \mathrm{II}\end{array}$ & $\begin{array}{l}21973.709 \\
21977.853 \\
21997.564 \\
22053.109 \\
22082.026\end{array}$ & $\begin{array}{l}a^{2} \mathrm{H}_{6}-a^{2} \mathrm{G}_{5} \\
a^{5} \mathrm{~F}_{3}-b^{5} \mathrm{~F}^{\prime}{ }_{2} \\
a^{5} \mathrm{~F}_{2}-b^{5} \mathrm{~F}^{\prime}{ }_{1} \\
a^{5} \mathrm{~F}_{5}-b^{5} \mathrm{~F}^{\prime} \\
a^{5} \mathrm{~F}_{1}-b^{5} \mathrm{~F}^{\prime}{ }_{2}\end{array}$ & \\
\hline $\begin{array}{l}4522.798 \\
4518.022 \\
4512.734 \\
4503.762 \\
4501.270\end{array}$ & $\begin{array}{r}30 \\
30 \\
30 \\
2 \\
23\end{array}$ & $\begin{array}{l}\mathrm{A} \\
\mathrm{A} \\
\mathrm{A} \\
\mathrm{D} \\
\mathrm{A}\end{array}$ & $\begin{array}{l}40 \mathrm{II} \\
50 \mathrm{II} \\
40 \mathrm{II} \\
4 n \mathrm{IV} \\
25 \mathrm{VE}, \mathrm{Ti} \mathrm{II}\end{array}$ & $\begin{array}{l}22104.030 \\
22127.394 \\
22153.325 \\
22197.454 \\
22209.745\end{array}$ & $\begin{array}{r}a^{5} \mathrm{~F}_{2}-b^{5} \mathrm{~F}^{\prime}{ }^{3} \\
a^{5} \mathrm{~F}_{3}-b^{5} \mathrm{~F}^{\prime}{ }_{4} \\
a^{5} \mathrm{~F}_{4}-b^{5} \mathrm{~F}^{\prime}{ }_{5} \\
a^{2} \mathrm{G}_{4}^{\prime}-a^{2} \mathrm{~F}^{\prime}{ }_{3}\end{array}$ & \\
\hline $\begin{array}{l}4496.146 \\
4489.089 \\
4482.688 \\
4481.261 \\
4474.852\end{array}$ & $\begin{array}{r}25 \\
25 \\
2 \\
26 \\
11\end{array}$ & $\begin{array}{l}\mathrm{A} \\
\mathrm{A} \\
\mathrm{D} \\
\mathrm{A} \\
\mathrm{A}\end{array}$ & $\begin{array}{l}20 \mathrm{III} \\
20 \mathrm{III} \\
10 \mathrm{III} \\
30 \mathrm{III} \\
8 \mathrm{III}\end{array}$ & $\begin{array}{l}22235.054 \\
22270.009 \\
22301.809 \\
22308.910 \\
22340.862\end{array}$ & $\begin{array}{l}a^{5} \mathrm{P}_{3}-a^{5} \mathrm{P}^{\prime}{ }^{\prime}{ }^{5}{ }^{5} \mathrm{P}_{2}-a^{5} \mathrm{P}^{\prime}{ }_{1} \\
b^{3} \mathrm{~F}_{4}-f^{3} \mathrm{~F}^{\prime}{ }_{3} \\
a^{5} \mathrm{P}_{3}-a^{5} \mathrm{P}^{\prime}{ }_{3}^{3} \mathrm{~F}_{3}-f^{3} \mathrm{~F}^{\prime}{ }_{2}\end{array}$ & $\begin{array}{l}-003 \\
+001\end{array}$ \\
\hline $\begin{array}{l}4471.238 \\
4468.493 \\
4465.807 \\
4457.428 \\
4455.321\end{array}$ & $\begin{array}{l}25 \\
25 \\
25 \\
29 \\
28\end{array}$ & $\begin{array}{l}\mathrm{B} \\
\mathrm{A} \\
\mathrm{A} \\
\mathrm{A} \\
\mathrm{A}\end{array}$ & $\begin{array}{l}20 \mathrm{III} \\
25 \mathrm{VE}, \mathrm{Ti} \mathrm{II} \\
20 \mathrm{III} \\
40 \mathrm{II} \\
30 \mathrm{II}\end{array}$ & $\begin{array}{l}22358.920 \\
22372.651 \\
22386.108 \\
22428.187 \\
22438.796\end{array}$ & $\begin{array}{c}a^{5} \mathrm{P}_{1}-a^{5} \mathrm{P}^{\prime}{ }^{\prime} \\
a^{2} \mathrm{C}_{5}^{\prime}{ }_{5}-a^{2} \mathrm{~F}^{\prime}{ }_{4} \\
a^{5} \mathrm{P}_{2}-a^{5} \mathrm{P}^{\prime}{ }^{3} \\
b^{3} \mathrm{~F}_{4}-f^{3} \mathrm{~F}^{\prime}{ }^{4} \\
b^{3} \mathrm{~F}_{3}-f^{3} \mathrm{~F}^{\prime}{ }_{3}\end{array}$ & $\begin{array}{l}-002 \\
-001\end{array}$ \\
\hline $\begin{array}{l}4453.708 \\
4453.312 \\
4450.896 \\
4449.143 \\
4443.802\end{array}$ & $\begin{array}{r}2 \\
17 \\
29 \\
30 \\
21\end{array}$ & $\begin{array}{l}\mathrm{D} \\
\mathrm{C} \\
\mathrm{C} \\
\mathrm{A} \\
\mathrm{A}\end{array}$ & $\begin{array}{l}20 \mathrm{III} \\
30 \mathrm{II} \\
25 \mathrm{III} \\
30 \mathrm{III} \\
25 \mathrm{VE} \text {, Ti II }\end{array}$ & $\begin{array}{l}22446.921 \\
22448.916 \\
22461.104 \\
22469.951 \\
22496.960\end{array}$ & $\begin{array}{r}a^{3} \mathrm{G}^{\prime}{ }_{3}-e^{3} \mathrm{G}_{3} \\
b^{3} \mathrm{~F}_{2}-f^{3} \mathrm{~F}^{\prime}{ }_{2} \\
a^{3} \mathrm{G}^{\prime}{ }_{4}-e^{3} \mathrm{G}_{4} \\
a^{3} \mathrm{G}^{\prime}-e^{3} \mathrm{G}_{5} \\
a^{2} \mathrm{D}^{\prime}{ }_{2}-a^{2} \mathrm{~F}^{\prime}{ }_{3}\end{array}$ & -009 \\
\hline $\begin{array}{l}4440.345 \\
4436.586\end{array}$ & $\begin{array}{r}20 \\
5\end{array}$ & $\underset{\mathrm{C}}{\mathrm{A}}$ & $\begin{array}{rl}10 & \mathrm{III} \\
4 \mathrm{III}\end{array}$ & $\begin{array}{l}22514.475 \\
22533.551\end{array}$ & $\begin{array}{l}a^{3} \mathrm{G}^{\prime}{ }_{3}-c \mathrm{~F}^{\prime} \\
a^{3} \mathrm{G}^{\prime}{ }_{4}-e^{3} \mathrm{G}_{5}\end{array}$ & \\
\hline 4434. 003 & 14 & C & $15 \mathrm{III} A$ & 22546.674 & $\begin{array}{c}b^{3} \mathrm{~F}_{2} \mathrm{~F}^{3}-\mathrm{G}^{3} \mathrm{G}_{3}^{3} \mathrm{~F}^{3} \mathrm{y}^{3} \\
\mathrm{~F}_{2}\end{array}$ & \\
\hline 4430.366 & 12 & C & $7 \mathrm{III} A$ & 22565.183 & $b^{3} \mathrm{~F}_{3}-f^{3} \overline{\mathrm{F}}_{4}^{\prime}{ }_{4}$ & -001 \\
\hline $\begin{array}{l}4427.098 \\
4426.054 \\
4422.823 \\
4421.754 \\
4417.274\end{array}$ & $\begin{array}{r}30 \\
11 \\
17 \\
9 \\
13\end{array}$ & $\begin{array}{l}\mathrm{A} \\
\mathrm{C} \\
\mathrm{A} \\
\mathrm{C} \\
\mathrm{B}\end{array}$ & $\begin{array}{l}40 \mathrm{III} \\
10 \mathrm{II} \\
10 \mathrm{II} \\
6 \mathrm{III} \\
15 \mathrm{III}\end{array}$ & $\begin{array}{l}22581.841 \\
22587.166 \\
22603.667 \\
22609.135 \\
22632.064\end{array}$ & $\begin{array}{c}a \mathrm{G}^{\prime}-b \mathrm{G} \\
a^{3} \mathrm{G}_{4}^{\prime}-g^{3} \mathrm{~F}^{\prime}{ }_{3} \\
a^{3} \mathrm{P}_{2}-f^{3} \mathrm{D}_{3} \\
b^{3} \mathrm{P}_{1}-j^{3} \mathrm{D}_{2} \\
a^{3} \mathrm{G}^{\prime}{ }_{5}-g^{3} \mathrm{~F}^{\prime}{ }_{4}\end{array}$ & -001 \\
\hline $\begin{array}{l}4399.767 \\
4395.031 \\
4393.925 \\
4372.383 \\
4359.682\end{array}$ & $\begin{array}{r}6 \\
26 \\
15 \\
2 \\
10\end{array}$ & $\begin{array}{l}\text { B } \\
\text { A } \\
\text { B } \\
\text { D } \\
\text { C }\end{array}$ & $\begin{array}{l}6 \mathrm{VE}, \mathrm{Ti} \mathrm{II} \\
25 \mathrm{VE} \text {, Ti II } \\
8 \mathrm{II} \\
3 \mathrm{IV} \\
5 n \mathrm{IV}\end{array}$ & $\begin{array}{l}22722.119 \\
22746.602 \\
22752.326 \\
22864.423 \\
22878.552\end{array}$ & $\begin{array}{c}a^{2} \mathrm{P}_{2}-a^{4} \mathrm{D}_{3} \\
a^{2} \mathrm{D}_{3}^{\prime}-a^{2} \mathrm{~F}_{4}{ }_{4} \\
b \mathrm{G}^{\prime}-f \mathrm{G} \\
a \mathrm{H}-g \mathrm{G}\end{array}$ & \\
\hline $\begin{array}{l}4360.487 \\
4346.104 \\
4337.916 \\
4325.134 \\
4321.655\end{array}$ & $\begin{array}{r}11 \\
11 \\
19 \\
6 \\
20\end{array}$ & $\begin{array}{l}\mathrm{B} \\
\mathrm{A} \\
\mathrm{A} \\
\mathrm{A} \\
\mathrm{A}\end{array}$ & $\begin{array}{l}4 \mathrm{III} \\
5 \mathrm{IV} \\
10 \mathrm{VE}, \mathrm{Ti} \mathrm{II} \\
9 n \mathrm{III} \\
8 n \mathrm{III}\end{array}$ & $\begin{array}{l}22925.798 \\
23002.667 \\
23046.089 \\
23114.194 \\
23132.801\end{array}$ & $\begin{array}{c}a^{3} \mathrm{D}_{3}^{\prime}-e^{3} \mathrm{P}^{\prime}{ }_{2} \\
a^{3} \mathrm{H}_{4}-f \mathrm{G} \\
a^{2} \mathrm{D}_{2}^{\prime}-a^{2} \mathrm{D}_{2} \\
a^{3} \mathrm{H}^{5}-f^{3} \mathrm{G}_{4} \\
a^{3} \mathrm{H}_{4}-f^{3} \mathrm{G}_{3}\end{array}$ & \\
\hline 4318. 631 & 25 & A & $10 n \mathrm{III}$ & 23149.000 & $a^{3} \mathrm{H}_{6}-f^{3} \mathrm{G}_{5}$ & \\
\hline 4314.801 & 30 & A & $25 \mathrm{II}$ & 23169. 542 & $\begin{array}{l}a^{5_{5}} 4-e^{3} \mathrm{D}_{3} \\
a^{5} \mathrm{~F}_{2}-e^{3} \mathrm{D}_{2}\end{array}$ & \\
\hline $\begin{array}{l}4312.861 \\
4307.900\end{array}$ & $\begin{array}{r}7 \\
21\end{array}$ & $\begin{array}{l}\mathrm{C} \\
\mathrm{B}\end{array}$ & $\begin{array}{l}7 \mathrm{VE}, \mathrm{Ti} \mathrm{II} \\
12 \mathrm{VE}, \mathrm{Ti} I \mathrm{II}\end{array}$ & $\begin{array}{l}23179.966 \\
23206.664\end{array}$ & $\begin{array}{l}a^{4} \mathrm{P}_{3}-a^{4} \mathrm{D}_{3} \\
a^{4} \mathrm{P}_{2}-a^{4} \mathrm{D}_{2}\end{array}$ & \\
\hline $\begin{array}{l}4305.910 \\
4298.664 \\
4295.751 \\
4294.101 \\
4289.068\end{array}$ & $\begin{array}{l}26 \\
29 \\
29 \\
11 \\
27\end{array}$ & $\begin{array}{l}\mathrm{A} \\
\mathrm{A} \\
\mathrm{A} \\
\mathrm{C} \\
\mathrm{A}\end{array}$ & $\begin{array}{l}60 \mathrm{II} \\
40 \mathrm{II} \\
22 \mathrm{II} \\
8 \mathrm{VE}, \mathrm{Ti} \mathrm{II} \\
25 \mathrm{II}\end{array}$ & $\begin{array}{l}\text { 23217. } 386 \\
23256.522 \\
23272.294 \\
23281.234 \\
23308.551\end{array}$ & $\begin{array}{r}a^{5} \mathrm{~F}_{5}-b^{5} \mathrm{D}_{4} \\
a^{5} \mathrm{~F}_{2}-b^{5} \mathrm{D}_{1} \\
a^{5} \mathrm{~F}_{1}-b^{5} \mathrm{D}_{0} \\
a^{2} \mathrm{D}_{3}-a^{2} \mathrm{D}_{3} \\
a^{5} \mathrm{~F}_{2}-b^{5} \mathrm{D}_{2}\end{array}$ & \\
\hline $\begin{array}{l}4287.405 \\
4286.006 \\
4282.702 \\
4281.371\end{array}$ & $\begin{array}{l}26 \\
30 \\
23 \\
11\end{array}$ & $\begin{array}{l}\mathrm{A} \\
\mathrm{A} \\
\mathrm{A} \\
\mathrm{C}\end{array}$ & $\begin{array}{l}22 \mathrm{II} \\
25 \mathrm{II} \\
12 \mathrm{III} \\
10 \mathrm{III}\end{array}$ & $\begin{array}{l}23317.595 \\
23325.204 \\
23343.199 \\
23350.459\end{array}$ & $\begin{array}{c}a^{5} \mathrm{~F}_{4}-b^{5} \mathrm{D}_{4} \\
a^{5} \mathrm{~F}_{3}-b_{3} D_{3} \\
a^{3} \mathrm{G}_{3}-h^{3} \mathrm{~F}_{2}^{\prime} \\
a^{5} \mathrm{~F}_{1}-b^{5} \mathrm{D}_{2}\end{array}$ & \\
\hline 4274.584 & 13 & B & $15 \mathrm{III}$ & 23387. 529 & $\left\{\begin{array}{r}a^{3} \mathrm{G}_{4}^{\prime}-h^{3} \mathrm{~F}^{\prime}{ }_{3}^{5} \\
a^{5} \mathrm{~F}_{2}-b^{5} \mathrm{D}_{3}\end{array}\right.$ & \\
\hline
\end{tabular}


TABLE 1.-Vacuum arc wave lengths of titanium-Continued

\begin{tabular}{|c|c|c|c|c|c|c|}
\hline$\lambda$ I. A. & $\begin{array}{c}\text { Num- } \\
\text { ber of } \\
\text { obser- } \\
\text { va- } \\
\text { tions }\end{array}$ & Weight & $\begin{array}{l}\text { Intensity and } \\
\text { temperature class }\end{array}$ & $\nu(\mathrm{Vac})$ & $\begin{array}{l}\text { Term combi- } \\
\text { nation }\end{array}$ & $(\mathrm{O}-\mathrm{C}) \lambda$ \\
\hline $\begin{array}{l}4263.134 \\
4258.523 \\
4256.025 \\
4249.114 \\
4237.889\end{array}$ & $\begin{array}{r}25 \\
3 \\
10 \\
5 \\
10\end{array}$ & $\begin{array}{l}\mathrm{A} \\
\mathrm{D} \\
\mathrm{B} \\
\mathrm{D} \\
\mathrm{A}\end{array}$ & $\begin{array}{l}15 \mathrm{III} \\
4 n \mathrm{IV} \\
8 n \mathrm{III} \\
5 n \mathrm{III} \\
7 \mathrm{III}\end{array}$ & $\begin{array}{l}23450.343 \\
23475.738 \\
23489.513 \\
23527.718 \\
23590.035\end{array}$ & $\begin{array}{c}a^{3} \mathrm{G}^{\prime}{ }_{5}-h^{3} \mathrm{~F}^{\prime}{ }_{4} \\
a^{5} \mathrm{D}_{1}-b^{5} \mathrm{D}^{\prime}{ }_{2} \\
a^{5} \mathrm{D}_{4}-b^{5} \mathrm{D}^{\prime}{ }_{4} \\
a^{5} \mathrm{D}_{2}-b^{5} \mathrm{D}_{3}^{\prime}{ }_{3} \\
b \mathrm{D}^{\prime}-d \mathrm{D}^{3}\end{array}$ & \\
\hline $\begin{array}{l}4186.119 \\
4171.897 \\
4163.644 \\
4159.634 \\
4150.963\end{array}$ & $\begin{array}{r}25 \\
8 \\
8 \\
11 \\
8\end{array}$ & $\begin{array}{l}\mathrm{A} \\
\mathrm{C} \\
\mathrm{C} \\
\mathrm{A} \\
\mathrm{C}\end{array}$ & $\begin{array}{l}25 \text { III } \\
5 \mathrm{VE}, \mathrm{Ti} \text { II } \\
8 \mathrm{VE}, \mathrm{Ti} \text { II } \\
9 \mathrm{III} \\
10 \mathrm{III}\end{array}$ & $\begin{array}{l}23881.766 \\
23963.179 \\
24010.682 \\
24033.828 \\
24084.030\end{array}$ & $\begin{array}{c}a \mathrm{G}^{\prime}-c \mathrm{G} \\
b^{2} \mathrm{~F}_{3}-c^{2} \mathrm{D}_{2} \\
b^{2} \mathrm{~F}_{4}-c^{2} \mathrm{D}_{3} \\
a^{3} \mathrm{D}^{\prime}{ }_{2}-i^{3} \mathrm{~F}^{\prime}{ }_{3} \\
a^{3} \mathrm{D}^{\prime}{ }_{3}-i^{3} \mathrm{~F}^{\prime}{ }_{4}\end{array}$ & \\
\hline $\begin{array}{l}4137.284 \\
4127.531 \\
4122.143 \\
4112.708 \\
4099.166\end{array}$ & $\begin{array}{r}10 \\
12 \\
7 \\
25 \\
8\end{array}$ & $\begin{array}{l}\mathrm{B} \\
\mathrm{B} \\
\mathrm{C} \\
\mathrm{A} \\
\mathrm{B}\end{array}$ & $\begin{array}{l}10 n \text { III } \\
15 \text { III } \\
10 \text { III } \\
20 \text { II } \\
8 \text { III }\end{array}$ & $\begin{array}{l}24163.654 \\
24220.752 \\
24252.407 \\
24308.047 \\
24388.346\end{array}$ & $\begin{array}{c}a^{5} \mathrm{D}_{4}-b^{5} \mathrm{P}_{3} \\
a^{3} \mathrm{G}_{5}-b^{3} \mathrm{H}_{6} \\
a^{3} \mathrm{G}_{3}-b^{3} \mathrm{H}_{4} \\
a^{3} \mathrm{~F}_{4}-a G \\
a^{3} \mathrm{D}^{\prime}{ }_{3}-f^{3} \mathrm{P}_{2}^{\prime}\end{array}$ & \\
\hline $\begin{array}{l}4082.456 \\
4078.471 \\
4065.094 \\
4060.263 \\
4058.139\end{array}$ & $\begin{array}{r}24 \\
26 \\
8 \\
23 \\
6\end{array}$ & $\begin{array}{l}\mathrm{A} \\
\mathrm{A} \\
\mathrm{B} \\
\mathrm{A} \\
\mathrm{D}\end{array}$ & $\begin{array}{l}20 \mathrm{III} \\
30 \mathrm{III} \\
15 \mathrm{III} \\
20 \mathrm{III} \\
7 \mathrm{IV}\end{array}$ & $\begin{array}{l}24488.173 \\
24512.099 \\
24592.756 \\
24622.015 \\
24634.904\end{array}$ & $\begin{array}{l}a^{3} \mathrm{P}_{2}-c^{3} \mathrm{P}^{\prime}{ }_{1} \\
a^{3} \mathrm{P}_{2}-c^{3} \mathrm{P}^{\prime}{ }_{2} \\
a^{3} \mathrm{P}_{1}-c^{3} \mathrm{P}^{\prime}{ }^{0} \\
a^{3} \mathrm{P}_{1}-c^{3} \mathrm{P}^{\prime}{ }_{2}\end{array}$ & \\
\hline $\begin{array}{l}4055.011 \\
4035.828 \\
4030.512 \\
4026.539 \\
4024.573\end{array}$ & $\begin{array}{r}22 \\
1 \\
15 \\
10 \\
29\end{array}$ & $\begin{array}{l}\mathrm{A} \\
\mathrm{D} \\
\mathrm{A} \\
\mathrm{B} \\
\mathrm{B}\end{array}$ & $\begin{array}{l}20 \text { III } \\
10 \text { III } \\
25 n \text { III } \\
25 n \text { III } \\
35 \text { II }\end{array}$ & $\begin{array}{l}24653.908 \\
24771.090 \\
24803.759 \\
24828.232 \\
24840.363\end{array}$ & $\begin{array}{c}a^{3} \mathrm{P}_{0}-c^{3} \mathrm{P}^{\prime}{ }_{1} \\
a^{3} \mathrm{D}^{\prime}{ }_{3}-k^{3} \mathrm{D}_{3} \\
a^{5} \mathrm{~F}^{\prime}{ }_{5}-b^{5} \mathrm{G}_{6}{ }_{6} \\
a^{5} \mathrm{~F}^{\prime}{ }_{4}-b^{5} \mathrm{G}^{\prime}{ }_{5} \\
a^{3} \mathrm{~F}_{4}-b^{3} \mathrm{~F}^{\prime}{ }_{3}\end{array}$ & 000 \\
\hline $\begin{array}{l}\text { 4017. } 771 \\
4015.377 \\
4013.587 \\
4008.926 \\
3998.635\end{array}$ & $\begin{array}{r}10 \\
8 \\
8 \\
28 \\
25\end{array}$ & $\begin{array}{l}\mathrm{B} \\
\mathrm{B} \\
\mathrm{B} \\
\mathrm{A} \\
\mathrm{A}\end{array}$ & $\begin{array}{l}15 n \text { III } \\
12 n \text { III } \\
12 n \text { III } \\
35 \text { II } \\
100 R \text { II }\end{array}$ & $\begin{array}{l}24882.418 \\
24897.249 \\
24908.356 \\
24937.314 \\
25001.494\end{array}$ & $\begin{array}{c}a^{5} \mathrm{~F}^{\prime}{ }_{2}-b^{5} \mathrm{G}^{\prime}{ }_{3} \\
a^{5} \mathrm{~F}^{\prime}{ }_{1}-b^{5} \mathrm{G}_{2}^{\prime} \\
a^{5} \mathrm{~F}_{5}{ }_{5}-b^{5} \mathrm{H}_{6} \\
a^{3} \mathrm{~F}_{3}-b^{3} \mathrm{~F}^{\prime}{ }_{2} \\
a^{3} \mathrm{~F}_{4}-b^{3} \mathrm{~F}_{4}^{\prime}\end{array}$ & $\begin{array}{l}+001 \\
-003\end{array}$ \\
\hline $\begin{array}{l}\text { 3989. } 758 \\
3981.761 \\
3964.269 \\
3962.851 \\
3958.206\end{array}$ & $\begin{array}{l}25 \\
26 \\
23 \\
23 \\
25\end{array}$ & $\begin{array}{l}\mathrm{A} \\
\mathrm{A} \\
\mathrm{B} \\
\mathrm{A} \\
\mathrm{B}\end{array}$ & $\begin{array}{l}80 R \text { II } \\
70 R \text { II } \\
35 \text { II } \\
35 \text { II } \\
80 \mathrm{II}\end{array}$ & $\begin{array}{l}25057.118 \\
25107.441 \\
25218.220 \\
25227.247 \\
25256.850\end{array}$ & $\begin{array}{l}a^{3} \mathrm{~F}_{3}-b^{3} \mathrm{~F}^{\prime}{ }^{3} \\
a^{3} \mathrm{~F}_{2}-b^{3} \mathrm{~F}^{\prime}{ }_{2} \\
a^{3} \mathrm{~F}_{3}-b^{3} \mathrm{~F}^{\prime}{ }_{4} \\
a^{3} \mathrm{~F}_{2}-b^{3} \mathrm{~F}^{\prime}{ }_{3} \\
a^{3} \mathrm{~F}_{4}-b^{3} \mathrm{D}_{3}\end{array}$ & $\begin{array}{l}-002 \\
+001 \\
-001 \\
-001 \\
+001\end{array}$ \\
\hline $\begin{array}{l}3956.336 \\
3948.670 \\
3947.770 \\
3929.875 \\
3924.527\end{array}$ & $\begin{array}{l}28 \\
26 \\
25 \\
25 \\
28\end{array}$ & $\begin{array}{l}\mathrm{A} \\
\mathrm{B} \\
\mathrm{B} \\
\mathrm{B} \\
\mathrm{A}\end{array}$ & $\begin{array}{l}60 \mathrm{II} \\
60 \mathrm{II} \\
40 \mathrm{II} \\
40 \mathrm{II} \\
50 \mathrm{II}\end{array}$ & $\begin{array}{l}25268.790 \\
25317.847 \\
25323.617 \\
25438.923 \\
25473.592\end{array}$ & $\begin{array}{l}a^{3} \mathrm{~F}_{3}-b^{3} \mathrm{D}_{2} \\
a^{3} \mathrm{~F}_{2}-b^{3} \mathrm{D}_{1} \\
a^{3} \mathrm{~F}_{3}-a^{3} \mathrm{P}^{\prime} 2 \\
a^{3} \mathrm{~F}_{2}-b^{3} \mathrm{D}_{2} \\
a^{3} \mathrm{~F}_{3}-b^{3} \mathrm{D}_{3}\end{array}$ & $\begin{array}{r}+002 \\
+004 \\
+001 \\
000\end{array}$ \\
\hline $\begin{array}{l}3921.423 \\
3914.334 \\
3913.464 \\
3904.785 \\
3900.546\end{array}$ & $\begin{array}{r}16 \\
20 \\
11 \\
26 \\
7\end{array}$ & $\begin{array}{l}\text { B } \\
\text { B } \\
\text { B } \\
\text { A } \\
\text { D }\end{array}$ & $\begin{array}{l}30 \mathrm{II} \\
35 \mathrm{II} \\
40 \mathrm{VE}, \mathrm{Ti} \mathrm{II} \\
40 n \mathrm{II} \\
50 \mathrm{VE}, \mathrm{Ti} \mathrm{II}\end{array}$ & $\begin{array}{l}25493.757 \\
25539.920 \\
25545.603 \\
25502.379 \\
25630.202\end{array}$ & $\begin{array}{c}a^{3} \mathrm{~F}_{2}-a^{3} \mathrm{P}^{\prime}{ }_{2} \\
a^{3} \mathrm{~F}_{4}-c^{3} \mathrm{~F}_{4}^{\prime} \\
a^{2} \mathrm{G}^{\prime}{ }_{4}-a^{2} \mathrm{G}_{4} \\
a \mathrm{D}^{\prime}-b \mathrm{~F}^{\prime} \\
a^{2} \mathrm{G}_{5}^{\prime}-a^{2} \mathrm{G}_{5}\end{array}$ & \\
\hline 3895.243 & 6 & C & $30 n$ III & 25665.092 & $a^{5} \mathrm{G}_{6}-b^{5} \mathrm{H}_{6}$ & \\
\hline 3875.262 & 14 & C & $20 n$ III & 25797.424 & $\begin{array}{l}a^{3} \mathrm{~F}_{2}-c^{3} \mathrm{~F}_{3} \\
a^{5} \mathrm{G}_{4}-b^{5} \mathrm{G}_{5}^{\prime}\end{array}$ & \\
\hline $\begin{array}{l}3866.446 \\
3798.276\end{array}$ & $\begin{array}{l}2 \\
3\end{array}$ & $\begin{array}{l}\mathrm{D} \\
\mathrm{D}\end{array}$ & $\begin{array}{l}15 n \mathrm{IV} \\
6 \mathrm{IV}\end{array}$ & $\begin{array}{l}25856.242 \\
26320.291\end{array}$ & $\begin{array}{l}a^{5} \mathrm{G}_{5}-b^{5} \mathrm{H}_{6} \\
b^{3} \mathrm{~F}_{2}-g^{3} \mathrm{D}_{1}\end{array}$ & \\
\hline $\begin{array}{l}3786.043 \\
3771.652 \\
3761.320 \\
3759.291 \\
3752.860\end{array}$ & $\begin{array}{l}23 \\
24 \\
24 \\
23 \\
24\end{array}$ & $\begin{array}{l}\mathrm{C} \\
\mathrm{A} \\
\mathrm{B} \\
\mathrm{B} \\
\mathrm{B}\end{array}$ & $\begin{array}{l}20 \mathrm{II} \\
25 \mathrm{I} \\
40 \mathrm{IVEr}, \mathrm{Ti} \mathrm{II} \\
40 \mathrm{IV} \mathrm{Er}, \mathrm{Ti} \mathrm{II} \\
80 \mathrm{r} \text { I }\end{array}$ & $\begin{array}{l}26405.331 \\
26506.075 \\
26578.885 \\
26593.234 \\
26638.799\end{array}$ & $\begin{array}{l}a \mathrm{D}^{\prime}-a \mathrm{P}^{\prime} \\
a^{3} \mathrm{~F}_{4}-d^{3} \mathrm{~F}^{\prime}{ }_{3} \\
a^{2} \mathrm{~F}_{3}-a^{2} \mathrm{~F}^{\prime}{ }_{3} \\
a^{2} \mathrm{~F}_{4}-a^{2} \mathrm{~F}^{\prime}{ }_{4} \\
a^{3} \mathrm{~F}_{4}-d^{3} \mathrm{~F}^{\prime}{ }_{4}\end{array}$ & $\begin{array}{r}000 \\
-001\end{array}$ \\
\hline $\begin{array}{l}3741.059 \\
3729.806 \\
3725.155 \\
3724.570 \\
3722.568\end{array}$ & $\begin{array}{r}25 \\
25 \\
5 \\
7 \\
14\end{array}$ & $\begin{array}{l}\text { B } \\
\text { A } \\
\text { C } \\
\text { C } \\
\text { B }\end{array}$ & $\begin{array}{l}60 r \text { I } \\
50 r \text { I } \\
20 \text { III } \\
20 \text { III } \\
15 \text { II }\end{array}$ & $\begin{array}{l}26722.827 \\
26803.450 \\
26836.920 \\
26841.134 \\
26855.565\end{array}$ & $\begin{array}{l}a^{3} \mathrm{~F}_{3}-d^{3} \mathrm{~F}^{\prime}{ }_{3} \\
a^{3} \mathrm{~F}_{2}-d^{3} \mathrm{~F}_{2}^{\prime} \\
a^{3} \mathrm{P}_{2}-b^{3} \mathrm{~S}_{1} \\
a \mathrm{G}^{\prime}-d \mathrm{G} \\
a^{3} \mathrm{~F}_{3}-d^{3} \mathrm{~F}_{4}^{\prime}\end{array}$ & $\begin{array}{l}-002 \\
+001\end{array}$ \\
\hline $\begin{array}{l}3717.393 \\
3694.445 \\
3689.916 \\
3685.192 \\
3671.672\end{array}$ & $\begin{array}{r}14 \\
9 \\
14 \\
17 \\
14\end{array}$ & $\begin{array}{l}\mathrm{A} \\
\mathrm{C} \\
\mathrm{A} \\
\mathrm{B} \\
\mathrm{A}\end{array}$ & $\begin{array}{l}20 \mathrm{I} \\
10 \mathrm{III} \\
15 \mathrm{I} \\
40 \mathrm{IV} \text { Er, Ti II } \\
20 \mathrm{I}\end{array}$ & $\begin{array}{l}26892.955 \\
27059.989 \\
27093.208 \\
27127.936 \\
27227.821\end{array}$ & $\begin{array}{l}a^{3} \mathrm{~F}_{2}-d^{3} \mathrm{~F}^{\prime} \\
b^{3} \mathrm{~F}_{3}-h^{3} \mathrm{D}_{2} \\
a^{3} \mathrm{~F}_{4}-c^{3} \mathrm{D}_{3} \\
a^{3} \mathrm{~F}_{4}-b^{3} \mathrm{G}_{4}\end{array}$ & $\begin{array}{r}-001 \\
+001 \\
000\end{array}$ \\
\hline
\end{tabular}


TABLE 1.-Vacuum arc wave lengths of titanium-Continued

\begin{tabular}{|c|c|c|c|c|c|c|}
\hline$\lambda I . A$. & $\begin{array}{l}\text { Num- } \\
\text { ber of } \\
\text { obser- } \\
\text { va- } \\
\text { tions }\end{array}$ & Weight & $\begin{array}{l}\text { Intensity and } \\
\text { temperature class }\end{array}$ & $\nu(\mathrm{Vac})$ & $\begin{array}{l}\text { Term combi- } \\
\text { nation }\end{array}$ & $(\mathrm{O}-\mathrm{C}) \lambda$ \\
\hline $\begin{array}{l}3668.965 \\
3660.631 \\
3658.097 \\
3654.592 \\
3653.497\end{array}$ & $\begin{array}{l}14 \\
12 \\
12 \\
10 \\
15\end{array}$ & $\begin{array}{l}\mathrm{B} \\
\mathrm{A} \\
\mathrm{B} \\
\mathrm{B} \\
\mathrm{C}\end{array}$ & $\begin{array}{l}15 \mathrm{I} \\
12 \mathrm{I} \\
20 \mathrm{I} \\
15 \mathrm{I} \\
100 r \mathrm{I}\end{array}$ & $\begin{array}{l}27247.912 \\
27309.943 \\
27328.863 \\
27355.073 \\
27363.270\end{array}$ & $\begin{array}{l}a^{3} \mathrm{~F}_{3}-c^{3} \mathrm{D}_{2} \\
a^{3} \mathrm{~F}_{3}-c^{3} \mathrm{D}_{3} \\
a^{3} \mathrm{~F}_{3}-b^{3} \mathrm{G}_{3} \\
a^{3} \mathrm{~F}_{2}-c^{3} \mathrm{D}_{1} \\
a^{3} \mathrm{~F}_{4}-b^{3} \mathrm{G}_{5}\end{array}$ & $\begin{array}{r}-001 \\
000 \\
+005 \\
-001 \\
+002\end{array}$ \\
\hline $\begin{array}{l}3646.198 \\
3642.675 \\
3641.330 \\
3635.462 \\
3624.826\end{array}$ & $\begin{array}{r}9 \\
14 \\
6 \\
12 \\
10\end{array}$ & $\begin{array}{l}\mathrm{A} \\
\mathrm{B} \\
\mathrm{B} \\
\mathrm{B} \\
\mathrm{B}\end{array}$ & $\begin{array}{l}12 \mathrm{I} \\
80 r \mathrm{I} \\
10 \mathrm{VE}, \mathrm{TiII} \\
80 r \mathrm{I} \\
8 \mathrm{VE}, \mathrm{Ti} I I\end{array}$ & $\begin{array}{l}27418.045 \\
27444.563 \\
27454.697 \\
27499.014 \\
27579.694\end{array}$ & $\begin{array}{l}a^{3} \mathrm{~F}_{2}-c^{3} \mathrm{D}_{2} \\
a^{3} \mathrm{~F}_{3}-b^{3} \mathrm{G}_{4} \\
a^{2} \mathrm{P}_{2}-a^{2} \mathrm{~S}_{1} \\
a^{3} \mathrm{~F}_{2}-b^{3} \mathrm{G}_{3} \\
a^{2} \mathrm{P}_{1}-a^{2} \mathrm{~S}_{1}\end{array}$ & $\begin{array}{r}-001 \\
000 \\
+003\end{array}$ \\
\hline $\begin{array}{l}3610.154 \\
3598.714 \\
3596.048 \\
3547.029 \\
3535.408\end{array}$ & $\begin{array}{r}9 \\
12 \\
9 \\
5 \\
9\end{array}$ & $\begin{array}{l}\mathrm{B} \\
\mathrm{A} \\
\mathrm{B} \\
\mathrm{B} \\
\mathrm{A}\end{array}$ & $\begin{array}{l}12 \mathrm{III} \\
15 \mathrm{III} \\
10 \mathrm{VE}, \mathrm{Ti} \mathrm{II} \\
15 \mathrm{IV} \\
10 \mathrm{VE}, \mathrm{Ti} \mathrm{II}\end{array}$ & $\begin{array}{l}27691.779 \\
27779.807 \\
27800.404 \\
28184.583 \\
28277.224\end{array}$ & $\begin{array}{l}a \mathrm{D}^{\prime}-b \mathrm{P}^{\prime} \\
a \mathrm{D}^{\prime}-c \mathrm{D} \\
a^{2} \mathrm{~F}_{4}-a^{4} \mathrm{D}_{3} \\
a \mathrm{G}^{\prime}-d \mathrm{~F}^{\prime} \\
b^{2} \mathrm{P}_{2}-c^{2} \mathrm{D}_{3}\end{array}$ & \\
\hline $\begin{array}{l}3510.840 \\
3504.890 \\
3491.053 \\
3480.525 \\
3477.181\end{array}$ & $\begin{array}{r}12 \\
12 \\
3 \\
5 \\
9\end{array}$ & $\begin{array}{l}\text { B } \\
\text { A } \\
\text { D } \\
\text { D } \\
\text { C }\end{array}$ & $\begin{array}{l}10 \mathrm{VEr}, \mathrm{Ti} \text { II } \\
8 \mathrm{VEr}, \mathrm{Ti} \text { II } \\
8 \mathrm{III} \text { Eru, Ti II } \\
12 \text { II } \\
15 \text { III Eru, Ti II }\end{array}$ & $\begin{array}{l}28475.101 \\
28523.436 \\
28636.491 \\
28723.103 \\
28750.726\end{array}$ & $\begin{array}{c}b^{2} \mathrm{G}^{\prime}{ }_{4}-b^{2} \mathrm{G}_{4} \\
b^{2} \mathrm{G}_{5}{ }_{5}-b^{2} \mathrm{G}_{5} \\
b^{4} \mathrm{~F}_{2}-a^{4} \mathrm{G}_{3} \\
a^{3} \mathrm{P}_{2}-d^{3} \mathrm{P}^{\prime}{ }_{2} \\
b^{4} \mathrm{~F}_{3}-a^{4} \mathrm{G}_{4}\end{array}$ & \\
\hline $\begin{array}{l}3461.496 \\
3444.306 \\
3394.574 \\
3387.834 \\
3385.944\end{array}$ & $\begin{array}{l}12 \\
12 \\
12 \\
12 \\
12\end{array}$ & $\begin{array}{l}\mathrm{A} \\
\mathrm{C} \\
\mathrm{B} \\
\mathrm{B} \\
\mathrm{B}\end{array}$ & $\begin{array}{l}20 \text { III Eru, Ti II } \\
15 \text { IIIEru, Ti II } \\
15 \text { IIIEru, Ti II } \\
15 \text { III Eru, Ti II } \\
40 r \text { II }\end{array}$ & $\begin{array}{l}\text { 28881. } 000 \\
29025.141 \\
29450.353 \\
29508.944 \\
29525.411\end{array}$ & $\begin{array}{l}b^{4} \mathrm{~F}_{4}-a^{4} \mathrm{G}_{5} \\
b^{4} \mathrm{~F}_{5}-a^{4} \mathrm{G}_{6} \\
a^{4} \mathrm{~F}_{3}-a^{4} \mathrm{G}_{3} \\
a^{4} \mathrm{~F}_{4}-a^{4} \mathrm{G}_{4} \\
a^{3} \mathrm{~F}_{4}-e^{3} \mathrm{D}_{3}\end{array}$ & 000 \\
\hline $\begin{array}{l}3383.761 \\
3380.278 \\
3377.577 \\
3371.447 \\
3370.436\end{array}$ & $\begin{array}{r}10 \\
12 \\
9 \\
4 \\
6\end{array}$ & $\begin{array}{l}\mathrm{A} \\
\mathrm{A} \\
\mathrm{C} \\
\mathrm{D} \\
\mathrm{C}\end{array}$ & $\begin{array}{l}40 \text { III Eru, Ti II } \\
15 \text { III Eru, Ti II } \\
30 r \text { I } \\
80 R \text { II } \\
40 r \text { II }\end{array}$ & $\begin{array}{l}29544.463 \\
29574.905 \\
29598.557 \\
29652.367 \\
29661.260\end{array}$ & $\begin{array}{l}a^{4} \mathrm{~F}_{2}-a^{4} \mathrm{G}_{3} \\
a^{4} \mathrm{~F}_{5}-a^{4} \mathrm{G}_{5} \\
a^{3} \mathrm{~F}_{3}-e^{3} \mathrm{D}_{2} \\
a^{3} \mathrm{~F}_{4}-c^{3} \mathrm{G}_{5} \\
a^{3} \mathrm{~F}_{2}-e^{3} \mathrm{D}_{1}\end{array}$ & $\begin{array}{l}+001 \\
+001 \\
+001\end{array}$ \\
\hline $\begin{array}{l}3361.213 \\
3358.271 \\
3354.634 \\
3349.399 \\
3341.875\end{array}$ & $\begin{array}{r}11 \\
5 \\
11 \\
11 \\
11\end{array}$ & $\begin{array}{l}\mathrm{B} \\
\mathrm{C} \\
\mathrm{B} \\
\mathrm{C} \\
\mathrm{B}\end{array}$ & $\begin{array}{l}40 \mathrm{IIIEr}, \mathrm{Ti} I \mathrm{II} \\
10 \mathrm{I} \\
60 r \mathrm{II} \\
40 \mathrm{II} E r, \mathrm{Ti} I I \\
50 r \mathrm{II}\end{array}$ & $\begin{array}{l}29742.652 \\
29768.709 \\
29800.975 \\
29847.557 \\
29914.755\end{array}$ & $\begin{array}{l}a^{4} \mathrm{~F}_{4}-a^{4} \mathrm{G}_{5} \\
a^{3} \mathrm{~F}_{2}-e^{3} \mathrm{D}_{2} \\
a^{3} \mathrm{~F}_{3}-c^{3} \mathrm{G}_{4} \\
a^{4} \mathrm{~F}_{5}-a^{4} \mathrm{G}_{6} \\
a^{3} \mathrm{~F}_{2}-c^{3} \mathrm{G}_{3}\end{array}$ & $\begin{array}{r}-002 \\
000 \\
+003\end{array}$ \\
\hline $\begin{array}{l}3340.344 \\
3335.192 \\
3332.111 \\
3329.455 \\
3326.762\end{array}$ & $\begin{array}{r}10 \\
12 \\
8 \\
9 \\
2\end{array}$ & $\begin{array}{l}\mathrm{C} \\
\mathrm{B} \\
\mathrm{B} \\
\mathrm{B} \\
\mathrm{D}\end{array}$ & $\begin{array}{l}15 \text { III Eru, Ti II } \\
20 \text { III Eru, Ti II } \\
8 \text { VEr, Ti II } \\
20 \text { III Eru, Ti II } \\
5 \text { III Eru, Ti II }\end{array}$ & $\begin{array}{l}29928.462 \\
29974.689 \\
30002.406 \\
30026.342 \\
30050.641\end{array}$ & $\begin{array}{l}b^{4} \mathrm{~F}_{2}-a^{4} \mathrm{~F}^{\prime}{ }_{2} \\
b^{4} \mathrm{~F}_{3}-a^{4} \mathrm{~F}^{\prime}{ }_{3} \\
b^{4} \mathrm{P}_{3}-a^{4} \mathrm{~S}_{2} \\
b^{4} \mathrm{~F}_{4}-a^{4} \mathrm{~F}^{\prime}{ }_{4} \\
b^{4} \mathrm{~F}_{2}-a^{4} \mathrm{~F}^{\prime}{ }_{3}\end{array}$ & \\
\hline $\begin{array}{l}3322.936 \\
3318.024 \\
3314.422 \\
3292.078 \\
3287.657\end{array}$ & $\begin{array}{r}11 \\
7 \\
10 \\
10 \\
10\end{array}$ & $\begin{array}{l}\mathrm{A} \\
\mathrm{A} \\
\mathrm{B} \\
\mathrm{B} \\
\mathrm{B}\end{array}$ & $\begin{array}{l}20 \text { III Eru, Ti II } \\
8 \text { III Eru, Ti II } \\
10 \mathrm{I} \\
20 \mathrm{I} \\
10 \mathrm{VEr}, \mathrm{Ti} I I\end{array}$ & $\begin{array}{l}30085.241 \\
30129.784 \\
30162.528 \\
30367.234 \\
30408.067\end{array}$ & $\begin{array}{c}b^{4} \mathrm{~F}_{5}-a^{4} \mathrm{~F}^{\prime}{ }_{5} \\
b^{4} \mathrm{~F}_{3}-a^{4} \mathrm{~F}^{\prime}{ }_{4} \\
a^{3} \mathrm{P}_{2}-h^{3} \mathrm{D}_{3} \\
a \mathrm{D}^{\prime}-c \mathrm{~F}^{\prime} \\
b^{2} \mathrm{G}^{\prime}{ }_{4}-a^{2} \mathrm{H}^{\prime}{ }_{5}\end{array}$ & \\
\hline 3261.596 & 8 & B & $25 \mathrm{VEr}, \mathrm{Ti} I \mathrm{I}$ & 30651.031 & $\left\{\begin{array}{c}b^{2} \mathrm{G}^{\prime}{ }_{5}-a^{2} \mathrm{H}^{\prime}{ }_{6} \\
b^{4} \mathrm{P}_{2}-b^{4} \mathrm{D}_{3}\end{array}\right.$ & \\
\hline $\begin{array}{l}3241.984 \\
3239.037 \\
3236.573\end{array}$ & $\begin{array}{l}10 \\
10 \\
10\end{array}$ & $\begin{array}{l}\mathrm{A} \\
\mathrm{C} \\
\mathrm{B}\end{array}$ & $\begin{array}{l}40 \mathrm{III} E r, \mathrm{Ti} \text { II } \\
40 \mathrm{III} E r, \mathrm{Ti} \text { II } \\
50 \mathrm{III} E r, \mathrm{Ti} \text { II }\end{array}$ & $\begin{array}{l}30836.445 \\
30864.588 \\
30888.089\end{array}$ & $\begin{array}{l}a^{4} \mathrm{~F}_{2}-a^{4} \mathrm{~F}^{\prime}{ }_{2} \\
a^{4} \mathrm{~F}_{3}-a^{4} \mathrm{~F}_{3}^{\prime} \\
a^{4} \mathrm{~F}_{4}-a^{4} \mathrm{~F}^{\prime}{ }_{4}\end{array}$ & \\
\hline $\begin{array}{l}3234.517 \\
3229.397 \\
3222.843 \\
3217.056 \\
3214.240\end{array}$ & $\begin{array}{r}10 \\
4 \\
9 \\
9 \\
8\end{array}$ & $\begin{array}{l}\mathrm{B} \\
\mathrm{D} \\
\mathrm{A} \\
\mathrm{A} \\
\mathrm{C}\end{array}$ & $\begin{array}{l}60 \mathrm{IIIEr}, \mathrm{Ti} \text { II } \\
10 \mathrm{VEru} \text {, Ti II } \\
15 \mathrm{IIIEr} \text { Ti II } \\
15 \text { III Er, Ti II } \\
12 \text { I }\end{array}$ & $\begin{array}{l}30907.726 \\
30956.623 \\
31019.583 \\
31075.376 \\
31102.603\end{array}$ & $\begin{array}{l}a^{4} \mathrm{~F}_{5}-a^{4} \mathrm{~F}^{\prime}{ }^{5} \\
a^{2} \mathrm{G}_{4}^{\prime}{ }_{4}-b^{2} \mathrm{~F}^{\prime}{ }_{4} \\
a^{4} \mathrm{~F}_{3}-a^{4} \mathrm{~F}^{\prime}{ }_{4} \\
a^{4} \mathrm{~F}_{4}-a^{4} \mathrm{~F}^{\prime}{ }_{5} \\
a^{3} \mathrm{~F}_{4}-d^{3} \mathrm{G}_{4}\end{array}$ & +002 \\
\hline $\begin{array}{l}3202.535 \\
3199.915 \\
3191.994 \\
3190.801 \\
3186.451\end{array}$ & $\begin{array}{r}9 \\
10 \\
9 \\
4 \\
10\end{array}$ & $\begin{array}{l}\text { A } \\
\text { B } \\
\text { A } \\
\text { D } \\
\text { A }\end{array}$ & $\begin{array}{l}12 \mathrm{VEr}, \mathrm{Ti} I I \\
100 R \mathrm{II} \\
80 R \mathrm{II} \\
20 \mathrm{IVEr}, \mathrm{Ti} I I \\
60 r \mathrm{II}\end{array}$ & $\begin{array}{l}31216.276 \\
31241.828 \\
31319.353 \\
31331.070 \\
31373.839\end{array}$ & $\begin{array}{r}a^{2} \mathrm{D}_{2}^{\prime}-b^{2} \mathrm{~F}^{\prime}{ }_{3} \\
a^{3} \mathrm{~F}_{4}-d^{3} \mathrm{G}_{5} \\
a^{3} \mathrm{~F}_{3}-d^{3} \mathrm{G}_{4} \\
a^{2} \mathrm{D}^{\prime}{ }_{3}-b^{2} \mathrm{~F}^{\prime}{ }_{4} \\
a^{3} \mathrm{~F}_{2}-d^{3} \mathrm{G}_{3}\end{array}$ & $\begin{array}{r}000 \\
000 \\
+002\end{array}$ \\
\hline $\begin{array}{l}3168.519 \\
3161.755 \\
3148.033 \\
3130.804 \\
3088.027\end{array}$ & $\begin{array}{r}10 \\
6 \\
2 \\
3 \\
10\end{array}$ & $\begin{array}{l}A \\
D \\
D \\
D \\
A\end{array}$ & $\begin{array}{l}30 \text { IIIEru, Ti II } \\
20 \text { IIIEru, Ti II } \\
12 \text { IVE, Ti II } \\
15 \text { IV, Ti II } \\
60 \text { IIIEr, Ti II }\end{array}$ & $\begin{array}{l}31551.385 \\
31618.884 \\
31756.698 \\
31931.457 \\
32373.764\end{array}$ & $\begin{array}{l}b^{4} \mathrm{~F}_{5}-a^{4} \mathrm{D}_{4} \\
b^{4} \mathrm{~F}^{3}-a^{4} \mathrm{D}_{2} \\
a^{4} \mathrm{~F}_{2}-a^{2} \mathrm{D}_{2} \\
a^{4} \mathrm{~F}_{3}-a^{2} \mathrm{D}_{3} \\
a^{4} \mathrm{~F}_{5}-a^{4} \mathrm{D}_{4}\end{array}$ & \\
\hline $\begin{array}{l}3078.645 \\
3075.225 \\
2956.133 \\
2948.255 \\
2941.995\end{array}$ & $\begin{array}{r}10 \\
10 \\
7 \\
8 \\
7\end{array}$ & $\begin{array}{l}\mathrm{A} \\
\mathrm{C} \\
\mathrm{C} \\
\mathrm{A} \\
\mathrm{C}\end{array}$ & $\begin{array}{l}45 \mathrm{IIIEr}, \mathrm{Ti} \text { II } \\
40 \mathrm{IIIEr}, \mathrm{Ti} \text { II } \\
70 R \mathrm{II} \\
60 r \mathrm{II} \\
60 r \mathrm{II}\end{array}$ & $\begin{array}{l}32472.424 \\
32508.527 \\
33818.127 \\
33908.489 \\
33980.630\end{array}$ & $\begin{array}{l}a^{4} \mathrm{~F}_{4}-a^{4} \mathrm{D}_{3} \\
a^{4} \mathrm{~F}_{3}-a^{4} \mathrm{D}_{2} \\
a^{3} \mathrm{~F}_{4}-f^{3} \mathrm{~F}^{\prime}{ }_{4} \\
a^{3} \mathrm{~F}_{3}-f^{3} \mathrm{~F}^{\prime}{ }_{3} \\
a^{3} \mathrm{~F}_{2}-f^{3} \mathrm{~F}^{\prime}{ }_{2}\end{array}$ & $\begin{array}{r}000 \\
000 \\
+004\end{array}$ \\
\hline
\end{tabular}




\section{COMPARISON WITH OTHER OBSERVATIONS}

Two other observers have measured wave lengths in the vacuum arc spectrum of titanium. In 1922 Brown ${ }^{13}$ published a list of more than 100 wave lengths between 4263 and $6261 \mathrm{~A}$ determined by interference methods in terms of the primary standard-the red ray of cadmium. This was followed two years later by a more extensive list by Crew ${ }^{14}$ who observed the region from 3653 to $6366 \mathrm{~A}$ with a plane grating giving high dispersion. There is most excellent agreement between the Bureau of Standards wave lengths and those of Brown in the interval from 4262 to $5200 \mathrm{~A}$. Most of the differences, Bureau of Standards minus Brown, are less than 0.003 A and exhibit no systematic shift between the two sets of results, the mean of the differences being $+0.0001 \mathrm{~A}$. Between 5200 and $5800 \mathrm{~A}$ Brown's list contains $13 \mathrm{Ti}$ lines, of which 11 may be compared with the $\mathrm{Bu}$ reau of Standards determinations. Of these, there are two positive differences, three zeros, and six negative differences, the mean value of Bureau of Standards minus Brown being -0.001 A. From 5800 to the end of Brown's list, his wave lengths are systematically longer than the Bureau of Standards values, the difference increasing from $-0.001 \mathrm{~A}$ at $5800 \mathrm{~A}$ to $-0.004 \mathrm{~A}$ at $5260 \mathrm{~A}$.

The comparison of the Bureau of Standards wave lengths with Crew's is limited to the region 3653 to $4263 \mathrm{~A}$, his wave lengths longer than $4263 \mathrm{~A}$ being based on those of Brown described above. The differences, Bureau of Standards minus Crew, change linearly from $-0.005 \mathrm{~A}$ at 3653 to $+0.002 \mathrm{~A}$ at $3800 \mathrm{~A}$, being zero at $3750 \mathrm{~A}$. From 3800 to $4000 \mathrm{~A}$ the difference is practically constant, amounting to $+0.002 \mathrm{~A}$. At $4000 \mathrm{~A}$ there is an abrupt increase in the difference to $+0.010 \mathrm{~A}$ from which it decreases linearly to $-0.010 \mathrm{~A}$ at $4263 \mathrm{~A}$, being zero in the vicinity of $4130 \mathrm{~A}$.

\section{COMPUTATION OF WAVE LENGTHS FROM SPECTRAL TERMS}

Classification of the lines of a spectrum as the difference of two spectral terms permits a calculation of the wave lengths of the lines if the term values are known. In recent years rapid progress has been made in the extension of our knowledge of the structure of spectra not only of neutral atoms which emit arc spectra, but also of ionized atoms which emit the various stages or orders of spark spectra. Such analyses of the spectrum of titanium have been made by Kiess and Kiess ${ }^{15}$ and by Russell. ${ }^{16}$ From the work of these

13 Astrophys. J., 56, p. 53; 1922.

${ }_{14}$ Astrophys. J., 60, p. 108; 1924.

15 J. Opt. Soc. Am. and Rev. Sci. Inst., 8, p. 607; 1924.

16 Astrophys. J. 66, pp. 283 and 347; 1927. 
investigators have been taken the term combinations or series classifications of the lines given in column 6 of Table 1 . The numerical values of the term combinations are the wave numbers in vacuum of the lines as entered in column 5.

If the terms involved in the production of the spectrum can be determined solely from lines measured in the yellow and red-that is, in the vicinity of the neon standards - then it is possible to calculate from them the wave lengths of lines in the blue and violet and thereby check the accuracy of observations remote from the standards. Such an ideal procedure, however, is not entirely possible in the case of titanium owing to the low intensities and consequent paucity of the longer Ti lines in the vacuum arc. An alternative procedure for testing the scale of a wave-length interval by means of cyclical term combinations, such as carried out by Meggers ${ }^{17}$ for the iron spectrum, was likewise not feasible for Ti because of the small number of cycles which could be formed from the wave lengths of Table 1. It was, therefore, decided to set up a system of terms based not only on the longer wave lengths of the table, but also on the relative term separations as given by observations throughout the measured wave-length interval. The degree of the constancy of these term separations is exhibited in Table 2, wherein are collected various differences used in calculating the relative values of the low terms.

TABLE 2.-Constancy of term differences throughout the spectrum

\begin{tabular}{|c|c|c|c|c|c|c|c|}
\hline$a^{3} \mathrm{~F}_{4}-a^{3} \mathrm{~F}_{3}$ & $a^{3} \mathrm{~F}_{3}-a^{3} \mathrm{~F}_{2}$ & $a^{3} \mathrm{~F}_{4}-b^{3} \mathrm{~F}_{4}$ & $a^{3} \mathrm{~F}_{3}-b^{3} \mathrm{~F}_{2}$ & $a^{3} \mathrm{~F}_{2}-b^{3} \mathrm{~F}_{2}$ & $a^{3} \mathrm{~F}_{4}-a^{3} \mathrm{P}_{2}$ & $a^{3} \mathrm{~F}_{3}-a^{3} \mathrm{P}_{1}$ & $a^{3} \mathbf{F}_{2}-a^{3} \mathrm{P}_{0}$ \\
\hline $\begin{array}{r}216.722 \\
.726 \\
.755 \\
.752 \\
.744\end{array}$ & $\begin{array}{r}170.122 \\
.129 \\
.129 \\
.127 \\
.106\end{array}$ & $\begin{array}{r}11389.934 \\
.960 \\
.955 \\
.952 \\
.940\end{array}$ & $\begin{array}{r}11469.688 \\
.672 \\
.686 \\
.701 \\
.693\end{array}$ & $\begin{array}{r}11531.789 \\
.786 \\
.800\end{array}$ & $\begin{array}{r}8215.486 \\
.488 \\
.467\end{array}$ & $\begin{array}{r}8322.307 \\
.310\end{array}$ & $\begin{array}{r}8436.646 \\
.597\end{array}$ \\
\hline $\begin{array}{l}.742 \\
.741 \\
.749 \\
.735\end{array}$ & $\begin{array}{l}.133 \\
.150 \\
.133 \\
.151 \\
.140\end{array}$ & $\begin{array}{l}.947 \\
.940\end{array}$ & .688 & & & & \\
\hline 216. 741 & 170.132 & 11389.947 & 11469.688 & 11531.792 & 8215.480 & 8322. 308 & 8436.622 \\
\hline
\end{tabular}

The mean values of the term differences of Table 2 were used in deriving the values of the terms $a^{3} \mathrm{~F}, b^{3} \mathrm{~F}$, and $a^{3} \mathrm{P}$ of Table 3 . From these the remainder of Table 3 was calculated by the joint use of wave numbers shorter than $21,300.000$ from Table 1 and relative term separations similar to those of Table 2. Although many of the well-observed lines of Table 1 belong to the singlet and quintet systems of Ti I, yet it was not possible to derive good term values for these systems by adhering to the limitations set for the derivation of the triplet system terms. The wave numbers calculated from

17 Astrophys. J., 60, p. 601924. 
these terms were converted into wave lengths which were compared with the observed values of Table 1 . The results of the comparison are shown in the last column of Table 1, where the residuals $\mathrm{O}-\mathrm{C}$ are entered. The algebraic sum of the differences $\mathrm{O}-\mathrm{C}$ for the interval $4700-2941 \mathrm{~A}$ is practically zero, although negative residuals predominate in the region $4700-3700 \mathrm{~A}$, and positive residuals in the region 3700-2941 A. However, since the wave lengths would require, in each of these regions, a mean correction of less than $0.001 \mathrm{~A}$ to reduce the sums of the residuals to zero, the representation of the wave lengths by means of the terms of Table 3 is regarded as satisfactory and is interpreted as indicating that the blue and violet lines have been measured to the same scale as those longer than $4700 \mathrm{~A}$. For lines longer than $4700 \mathrm{~A}$ the majority of the residuals are necessarily zero, since the lines were used directly in the computation of the terms. With one exception the residuals greater than $0.002 \mathrm{~A}$ in this region refer to lines observed only a few times.

TABLE 3.-Terms in the triplet system of titanium

\begin{tabular}{|c|c|c|c|c|c|c|c|c|c|}
\hline$a^{3} \mathrm{~F}_{4}$ & 386.873 & $a^{3} \mathrm{D}_{3}$ & 20126.072 & $a^{3} \mathrm{~F}_{4}^{\prime}$ & 19573. 980 & $a^{3} \mathrm{G}_{5}$ & 21739. 743 & $a^{3} \mathrm{H}_{6}^{\prime}$ & 32013. 555 \\
\hline$a^{3} \mathrm{~F}_{3}$ & 170.132 & $a^{3} \mathrm{D}_{2}$ & 20006. 049 & $a^{3} \mathrm{~F}^{\prime}{ }_{3}$ & 19421. 580 & $a^{3} \mathrm{G}^{4}$ & 21588. 520 & $a^{3} \mathrm{H}_{5}^{\prime}$ & 31914. 304 \\
\hline$a^{3} \mathrm{~F}_{2}$ & $\begin{array}{r}170.132 \\
0.000\end{array}$ & $a^{3} \mathrm{D}_{1}$ & $\begin{array}{r}08.171 \\
1937.878\end{array}$ & $a^{3} \mathrm{~F}_{2}^{\prime}$ & $\begin{array}{r}\text { 98. } 577 \\
19323.003\end{array}$ & $a^{3} \mathrm{G}_{3}$ & $\begin{array}{r}118.986 \\
21469.534\end{array}$ & $a^{3} \mathrm{H}^{\prime}{ }_{3}$ & 31830.016 \\
\hline$b^{3} \mathrm{~F}_{4}$ & $\begin{array}{r}11776.820 \\
137.000\end{array}$ & $b^{3} \mathrm{D}_{3}$ & $\begin{array}{r}25643.724 \\
204.794\end{array}$ & $b^{3} \mathrm{~F}_{4}^{\prime}$ & $\begin{array}{r}25388.345 \\
161.109\end{array}$ & $b^{3} \mathrm{G}_{5}$ & $\begin{array}{r}27750.156 \\
135.463\end{array}$ & $b^{3} \mathrm{H}_{6}^{\prime}$ & $\begin{array}{r}35685.188 \\
125.526\end{array}$ \\
\hline$b^{3} \mathrm{~F}_{3}$ & $\begin{array}{r}11639.820 \\
\end{array}$ & $b^{3} \mathrm{D}_{2}$ & $\begin{array}{r}25438.930 \\
121.088\end{array}$ & $b^{3} \mathrm{~F}^{\prime}{ }_{3}$ & $\begin{array}{r}25227.236 \\
119.783\end{array}$ & $b^{3} \mathrm{G}_{4}$ & $\begin{array}{r}27614.693 \\
115.660\end{array}$ & $b^{3} \mathrm{H}_{5}^{\prime}$ & 35559. 662 \\
\hline$b^{3} \mathrm{~F}_{2}$ & 11531.812 & $b^{3} \mathrm{D}_{1}$ & 25317.842 & $b^{3} \mathrm{~F}_{2}^{\prime}$ & 25107.453 & $b^{3} \mathrm{G}_{3}$ & 27499.033 & $b^{3} \mathrm{H}^{\prime}{ }_{4}$ & 35454.099 \\
\hline$a^{3} \mathrm{P}_{2}$ & $\begin{array}{r}860 \approx .353 \\
109 . \varepsilon \% 5\end{array}$ & $c^{3} \mathrm{D}_{3}$ & $\begin{array}{r}27480.077 \\
62.040\end{array}$ & $d^{3} \mathrm{~F}_{4}^{\prime}$ & $\begin{array}{r}27025.667 \\
132.721\end{array}$ & $c^{3} \mathrm{G}_{5}$ & $\begin{array}{r}30039.246 \\
68.140\end{array}$ & & \\
\hline$a^{3} \mathrm{P}_{1}$ & $\begin{array}{r}8492.4 \mathrm{i} \\
55.80 \%\end{array}$ & $c^{3} \mathrm{D}_{2}$ & $\begin{array}{r}27418.037 \\
62.972\end{array}$ & $d^{3} \mathrm{~F}^{\prime}{ }_{3}$ & $\begin{array}{r}26892.946 \\
89.484\end{array}$ & $c^{3} \mathrm{G}_{4}$ & $\begin{array}{r}29971.106 \\
56.333\end{array}$ & & \\
\hline$a^{3} \mathrm{P}_{0}$ & 8436.630 & $c^{3} \mathrm{D}_{1}$ & 27355.065 & $d^{3} \mathrm{~F}_{2}^{\prime}$ & 26803.462 & $c^{3} \mathrm{G}_{3}$ & 29914. 773 & & \\
\hline$a^{3} \mathrm{G}_{5}^{\prime}$ & $\begin{array}{r}15220.400 \\
63.597\end{array}$ & $c^{3} \mathrm{D}_{3}$ & $\begin{array}{r}29912.292 \\
143.606\end{array}$ & $e^{3} \mathrm{~F}_{4}$ & $\begin{array}{r}33700.897 \\
20.735\end{array}$ & $d^{3} \mathrm{G}_{5}$ & $\begin{array}{r}31628.698 \\
139.212\end{array}$ & & \\
\hline$a^{3} \mathrm{G}_{4}^{\prime}$ & 15156.803 & $e^{3} \mathrm{I}_{2}$ & 29768. 686 & $e^{3} \mathrm{~F}_{3}$ & 33680.162 & $d^{3} \mathrm{G}_{4}$ & 31489.486 & & \\
\hline$a^{3} \mathrm{G}^{\prime}{ }_{3}$ & 15108.153 & $e^{3} \mathrm{D}_{1}$ & 29661. 272 & $e^{3} \mathrm{~F}_{2}^{\prime}$ & 33655.898 & $d^{3} \mathrm{G}_{3}$ & 31373. 862 & & \\
\hline$a^{3} \mathrm{H}_{6}$ & 18192. 594 & $f^{3} \mathrm{D}_{3}$ & 31206. 014 & $f^{3} \mathrm{~F}_{4}$ & $\begin{array}{r}34205.001 \\
126.389\end{array}$ & & & & \\
\hline$a^{3} \mathrm{H}_{5}$ & 18141.252 & $f^{3} \mathrm{D}_{2}$ & 31190.663 & $f^{3} \mathrm{~F}^{\prime}$ & 34078.612 & & & & \\
\hline$a^{3} \mathrm{H}_{4}$ & & $f^{3} \mathrm{D}_{1}$ & $\begin{array}{r}0.0184 \\
31184.089\end{array}$ & $f^{3} \mathrm{~F}^{\prime}$ & 33980.685 & & & & \\
\hline
\end{tabular}

\section{RESULTS FOR OTHER ELEMENTS}

In Tables 4, 5, and 6 which follow are given the vacuum are wave lengths of a few lines of elements which appeared as impurities in the titanium or were especially observed to supply additional data for the solar work. The most abundant impurity in the titanium was iron; in lesser amounts were calcium, aluminum, chromium, vanadium, and sodium. The elements especially observed for wave lengths in the yellow and red were calcium, barium, manganese, and nickel, the observations being made at the same time as the first series of titanium. Owing to the fact that a copper rod was used 
nearly always for the negative electrode, 26 lines of this element were also measured on the spectrograms of all three series and are given below.

TABLE 4.-Vacuum arc wave lengths of iron

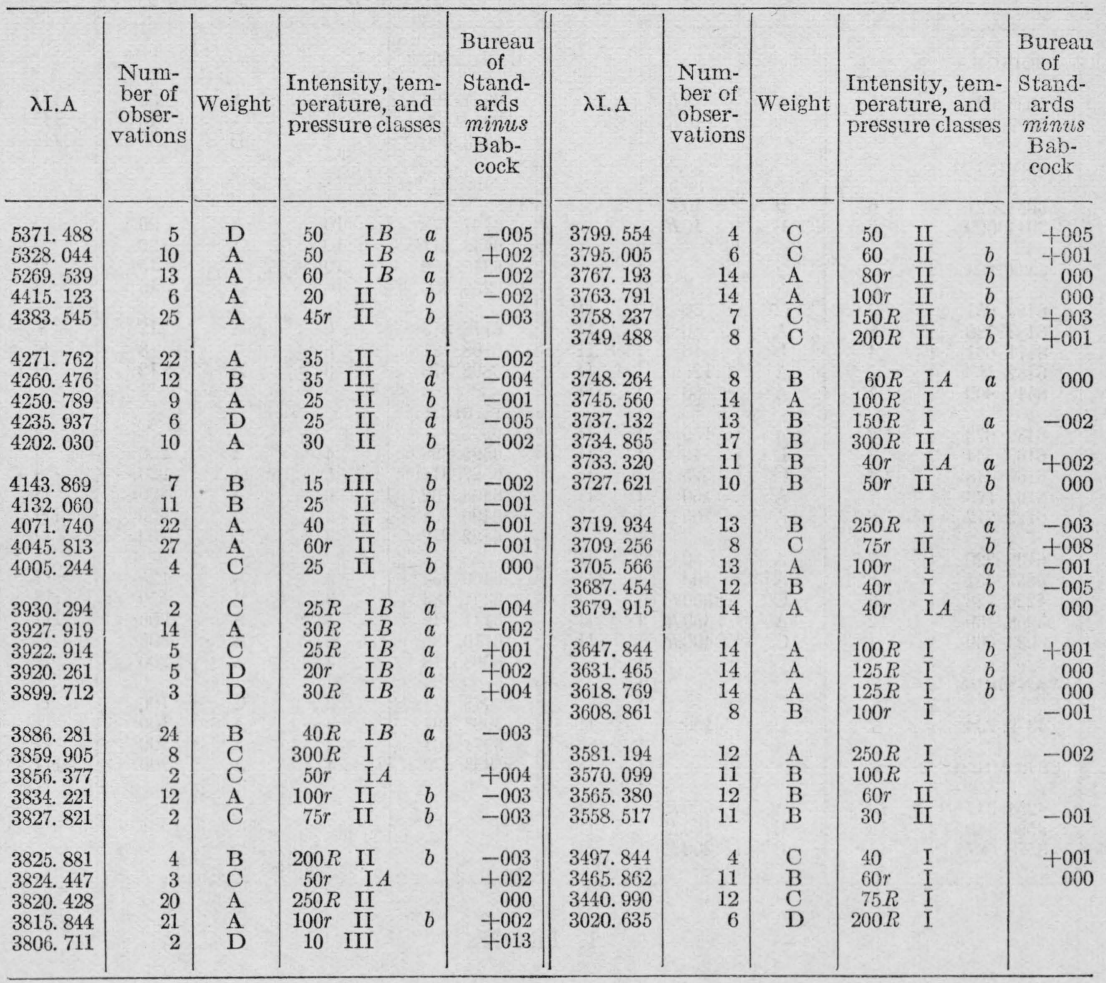

TABLE 5.-Vacuum arc wave lengths of copper

\begin{tabular}{|c|c|c|c|c|c|c|c|c|c|c|c|}
\hline \multirow{2}{*}{$\lambda$ I. A. } & \multirow{2}{*}{$\begin{array}{l}\text { Num- } \\
\text { ber of } \\
\text { obser- } \\
\text { vations }\end{array}$} & \multirow[t]{2}{*}{ Weight } & \multirow{2}{*}{$\begin{array}{l}\text { Inten- } \\
\text { sities }\end{array}$} & \multicolumn{2}{|c|}{$\begin{array}{l}\text { Bureau of } \\
\text { Standards } \\
\text { minus Wolf- } \\
\text { sohn }\end{array}$} & \multirow[t]{2}{*}{$\lambda$ I. A. } & \multirow{2}{*}{$\begin{array}{l}\text { Num- } \\
\text { ber of } \\
\text { obser- } \\
\text { vations }\end{array}$} & \multirow[t]{2}{*}{ Weight } & \multirow{2}{*}{$\begin{array}{l}\text { Inten- } \\
\text { sities }\end{array}$} & \multicolumn{2}{|c|}{$\begin{array}{l}\text { Bureau of } \\
\text { Standards } \\
\text { minus Wolf- } \\
\text { sohn }\end{array}$} \\
\hline & & & & $\begin{array}{l}\text { Vac- } \\
\text { uum }\end{array}$ & Air & & & & & $\begin{array}{l}\text { Vac- } \\
\text { uum }\end{array}$ & Air \\
\hline $\begin{array}{l}5782.132^{*} \\
5700.239^{*} \\
5292.519 \\
5220.070^{*} \\
5218.202\end{array}$ & $\begin{array}{r}31 \\
23 \\
9 \\
13 \\
27\end{array}$ & $\begin{array}{l}\mathrm{A} \\
\mathrm{A} \\
\mathrm{C} \\
\mathrm{A} \\
\mathrm{A}\end{array}$ & $\begin{array}{c}8 r \\
8 r \\
6 \\
6 \\
10\end{array}$ & $\begin{array}{l}-004 \\
-040 \\
-031\end{array}$ & $\begin{array}{r}-008 \\
000\end{array}$ & $\begin{array}{l}3530.382 \\
3337.845 \\
3279.815 \\
3273.956^{*}\end{array}$ & $\begin{array}{r}9 \\
10 \\
10 \\
7\end{array}$ & $\begin{array}{l}\mathrm{B} \\
\mathrm{A} \\
\mathrm{B} \\
\mathrm{A}\end{array}$ & $\begin{array}{c}7 \\
8 \\
5 \\
10 R\end{array}$ & $\begin{array}{l}-017 \\
-018\end{array}$ & $\begin{array}{l}-005 \\
-005\end{array}$ \\
\hline $\begin{array}{l}5153.237^{*} \\
5105.542 \\
4704.593 \\
4651.124 \\
4530.786^{*}\end{array}$ & $\begin{array}{r}27 \\
23 \\
6 \\
14 \\
11\end{array}$ & $\begin{array}{l}\mathrm{A} \\
\mathrm{A} \\
\mathrm{D} \\
\mathrm{B} \\
\mathrm{A}\end{array}$ & $\begin{array}{l}8 \\
7 \\
4 \\
8 \\
6\end{array}$ & $\begin{array}{l}-035 \\
-017 \\
-025 \\
-009\end{array}$ & $\begin{array}{l}+004 \\
-009\end{array}$ & $\begin{array}{l}3247.540^{*} \\
3208.230 \\
3194.096 \\
3063.411 \\
3036.101\end{array}$ & $\begin{array}{r}8 \\
8 \\
8 \\
10\end{array}$ & $\begin{array}{l}\mathrm{B} \\
\mathrm{B} \\
\mathrm{B} \\
\mathrm{A}\end{array}$ & $\begin{array}{c}10 R \\
6 \\
8 \\
7\end{array}$ & $\begin{array}{l}-016 \\
-020 \\
-018 \\
-015 \\
-013\end{array}$ & $\begin{array}{l}-002 \\
-010 \\
-008 \\
-006\end{array}$ \\
\hline $\begin{array}{l}4509.374 \\
4480.330^{*} \\
4062.639 \\
4022.627\end{array}$ & $\begin{array}{r}10 \\
6 \\
8 \\
11\end{array}$ & $\begin{array}{l}\text { B } \\
\text { C } \\
\text { C } \\
\text { A }\end{array}$ & $\begin{array}{r}6 \\
7 \\
10 \\
10\end{array}$ & $\begin{array}{l}-015 \\
-004\end{array}$ & -013 & $\begin{array}{l}3010.838 \\
2961.164 \\
2824.369\end{array}$ & $\begin{array}{r}10 \\
4 \\
4\end{array}$ & $\begin{array}{l}\mathrm{A} \\
\mathrm{D} \\
\mathrm{B}\end{array}$ & $\begin{array}{r}7 \\
9 \\
10\end{array}$ & $\begin{array}{l}-013 \\
-015 \\
-013\end{array}$ & $\begin{array}{l}-007 \\
-009\end{array}$ \\
\hline
\end{tabular}


TABLE 6.-Vacuum arc wave lengths of $\mathrm{Na}, \mathrm{Al}, \mathrm{Ca}, \mathrm{V}, \mathrm{Cr}, \mathrm{Mn}, \mathrm{Ni}$, and $\mathrm{Ba}$

\begin{tabular}{|c|c|c|c|c|c|c|c|c|c|}
\hline$\lambda$ I. A. & 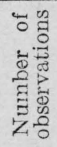 & 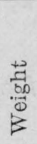 & 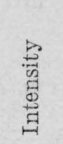 & 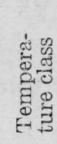 & $\lambda$ I. A. &  & $\begin{array}{l}\overrightarrow{0} \\
\text { b0 } \\
\overrightarrow{0}\end{array}$ & $\begin{array}{l}\text { 壭 } \\
\text { 离 } \\
\text { 苟 }\end{array}$ & 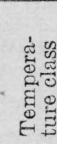 \\
\hline SODIUM & & & & & MANGANESE & & & & \\
\hline $\begin{array}{c}5895.927 \\
5889.954 \\
\text { ALUMINUM }\end{array}$ & $\begin{array}{l}5 \\
8\end{array}$ & $\stackrel{\mathrm{A}}{\mathrm{A}}$ & $\begin{array}{r}8 R \\
10 R\end{array}$ & & $\begin{array}{l}6021.798 \\
6016.639 \\
6013.490\end{array}$ & $\begin{array}{r}12 \\
9 \\
4\end{array}$ & $\begin{array}{l}\text { A } \\
\text { A } \\
\text { B }\end{array}$ & $\begin{array}{l}50 \\
40 \\
30\end{array}$ & $\begin{array}{l}\text { III } \\
\text { III } \\
\text { III }\end{array}$ \\
\hline $\begin{array}{l}\text { 3961. } 527 \\
\text { 3944. } 009 \\
\text { CAI.CIUM }\end{array}$ & $\begin{array}{l}6 \\
6\end{array}$ & $\begin{array}{l}\mathrm{B} \\
\mathrm{B}\end{array}$ & $\begin{array}{l}10 R \\
10 R\end{array}$ & & $\begin{array}{l}6767.778 \\
6643.641 \\
6314.666 \\
6256.365\end{array}$ & $\begin{array}{l}10 \\
10 \\
10 \\
10\end{array}$ & $\begin{array}{l}\mathrm{A} \\
\mathrm{A} \\
\mathrm{A} \\
\mathrm{A}\end{array}$ & $\begin{array}{l}20 \\
20 \\
15 \\
15\end{array}$ & $\begin{array}{l}\text { I } \\
\text { I } \\
\text { II } \\
\text { I }\end{array}$ \\
\hline $\begin{array}{l}6499.651 \\
6493.780 \\
6471.661 \\
6462.565 \\
6449.809\end{array}$ & $\begin{array}{l}3 \\
8 \\
4 \\
7 \\
7\end{array}$ & $\begin{array}{l}\text { C } \\
\text { A } \\
\text { B } \\
\text { A } \\
\text { A }\end{array}$ & $\begin{array}{r}30 \\
80 \\
40 \\
125 \\
50\end{array}$ & $\begin{array}{l}\text { II } \\
\text { II } \\
\text { II } \\
\text { II }\end{array}$ & $\begin{array}{l}6176.813 \\
6108.121 \\
5892.878 \\
\end{array}$ & $\begin{array}{l}6 \\
6 \\
6\end{array}$ & $\begin{array}{l}\mathrm{A} \\
\mathrm{A} \\
\mathrm{A}\end{array}$ & $\begin{array}{r}12 \\
8 \\
12\end{array}$ & $\begin{array}{l}\text { V } \\
\text { II } \\
\text { II }\end{array}$ \\
\hline $\begin{array}{l}6439.073 \\
6169.554 \\
6169.048 \\
6162.173 \\
6122.217\end{array}$ & $\begin{array}{l}7 \\
3 \\
4 \\
7 \\
8\end{array}$ & $\begin{array}{l}\text { A } \\
\text { B } \\
\text { C } \\
\text { A } \\
\text { A }\end{array}$ & $\begin{array}{r}150 \\
40 \\
25 \\
150 \\
100\end{array}$ & $\begin{array}{l}\text { II } \\
\text { III } \\
\text { III } \\
\text { II } \\
\text { II }\end{array}$ & $\begin{array}{l}6595.328 \\
6527.314 \\
6498.762 \\
6496.901 \\
6482.912\end{array}$ & $\begin{array}{l}4 \\
5 \\
6 \\
6 \\
3\end{array}$ & $\begin{array}{l}\mathrm{A} \\
\mathrm{A} \\
\mathrm{A} \\
\mathrm{A} \\
\mathrm{B}\end{array}$ & $\begin{array}{l}200 \\
250 \\
300 r \\
600 r \\
200\end{array}$ & $\begin{array}{r}\text { I } \\
\text { II } \\
\text { III } \\
\text { II }\end{array}$ \\
\hline $\begin{array}{l}6102.720 \\
5857.451 \\
4226.728 \\
3968.469 \\
3933.669\end{array}$ & $\begin{array}{r}7 \\
6 \\
3 \\
12 \\
4\end{array}$ & $\begin{array}{l}\text { A } \\
\text { A } \\
\text { C } \\
\text { A } \\
\text { C }\end{array}$ & $\begin{array}{l}80 \\
100 \\
500 R \\
350 R \\
400 R\end{array}$ & $\begin{array}{l}\text { II } \\
\text { III } \\
\text { II } \\
\text { II }\end{array}$ & $\begin{array}{l}6450.854 \\
6341.683 \\
6141.716 \\
6110.785 \\
6063.118\end{array}$ & $\begin{array}{l}3 \\
3 \\
5 \\
5 \\
3\end{array}$ & $\begin{array}{l}\mathrm{A} \\
\mathrm{A} \\
\mathrm{A} \\
\mathrm{A} \\
\mathrm{A}\end{array}$ & $\begin{array}{l}125 \\
150 \\
600 r \\
300 r \\
200\end{array}$ & $\begin{aligned} \text { I } \\
\text { II } \\
\text { II } \\
\text { II }\end{aligned}$ \\
\hline $\begin{array}{l}\text { VANADIUM } \\
4379.234 \\
\text { CHROMIUM }\end{array}$ & 7 & B & $150 r$ & II & $\begin{array}{l}6019.474 \\
5997.091 \\
5971.701 \\
5853.679\end{array}$ & $\begin{array}{l}2 \\
3 \\
3 \\
4\end{array}$ & $\begin{array}{l}\mathrm{C} \\
\mathrm{A} \\
\mathrm{A} \\
\mathrm{A}\end{array}$ & $\begin{array}{l}100 \\
100 \\
100 \\
200\end{array}$ & $\begin{array}{l}\text { II } \\
\text { II } \\
\text { II } \\
\text { III }\end{array}$ \\
\hline $\begin{array}{l}4254.337 \\
3593.488 \\
3578.687\end{array}$ & $\begin{array}{l}19 \\
12 \\
11\end{array}$ & $\begin{array}{l}A \\
B \\
B\end{array}$ & $\begin{array}{l}500 R \\
160 R \\
200 R\end{array}$ & $\begin{array}{l}\text { II } \\
\text { II }\end{array}$ & & & & & \\
\hline
\end{tabular}

\section{IRON}

The iron lines whose wave lengths are given in the first column of Table 4 all originate in the lowest or the low metastable states of the iron atom. They are, accordingly, easily excited and appear with great intensity. In ares in air most of them are reversed. The character of the lines in arcs in air and their behavior in the vacuum furnace are described in columns 4 and 5 , the data for which are taken from King. ${ }^{18}$ In the vacuum arc, however, these lines become narrow and give sharp interference fringes with high orders, a phenomenon pointed out long ago by Fabry and Buisson. ${ }^{19}$ With two exceptions these lines belong to pressure classes $a$ and $b$ which means that they experience the least displacement with increasing pressure. We should, therefore, expect to find very little difference between their wave lengths in vacuum and in air, the amount of the displacement increasing with wave length. Such, indeed, is the case. Quite

${ }^{18}$ Astrophys. J., 37, p. 239; 1913; 56, p. 318; 1922.

10 J. de Physique (IV), 9, p. 947; 1910. 
recently Babcock ${ }^{20}$ has published interferometer measurements of iron wave lengths emitted by ares in air. The comparison of the Bureau of Standards values with his is shown in the last column of Table 4. In the ultra-violet - that is, the region of wave lengths shorter than 3922 A-the residuals, Bureau of Standards minus Babcock, are about equally distributed among positive and negative values, being in the mean $+0.0007 \mathrm{~A}$. But for all lines longer than $3922 \mathrm{~A}$ the residuals, Bureau of Standards minus Babcock, are negative, with one exception, the mean being $-0.002 \mathrm{~A}$.

\section{COPPER}

The copper lines which were measured are listed in Table 5. Of these, the pair at 5700 and $5782 \mathrm{~A}$ in the yellow, and the raies ultimes, 3247 and $3274 \mathrm{~A}$, always appeared reversed. The intensities assigned to the lines are taken from Kayser's Tabelle der Hauptlinien. Four pairs of lines in this table, marked with an asterisk (*), involve the term ${ }^{2} \mathrm{P}_{1},_{2}$ as may be seen by reference to Shenstone's ${ }^{21}$ classification of the $\mathrm{Cu}$ arc spectrum. They may, therefore, be used to determine the separation of the components of this term, and thereby check the relative accuracy of the measurements. The four values of ${ }^{2} \mathrm{P}_{2}-{ }^{2} \mathrm{P}_{1}$ are: $248.392,248.377,248.335$, and 248.364 . The low value of the third is accounted for by the fact that the wave length $4480.360 \mathrm{~A}$ which enters into its determination is affected by the Ti line at 4480.60. A correction of $-0.009 \mathrm{~A}$ applied to the tabulated wave length would give a value of ${ }^{2} \mathrm{P}_{2}-{ }^{2} \mathrm{P}_{1}$ equal to the mean of the other three determinations.

The vacuum arc spectrum of $\mathrm{Cu}$ has been measured recently by Wolfsohn ${ }^{22}$ who photographed it in juxtaposition with the spectrum of the are in air, using the high dispersion afforded by the grating spectrograph at Bonn. His vacuum values are systematically larger than his air values which he interprets as a pressure shift toward the violet for lines emitted by the arc in air. A comparison of the Bureau of Standards wave lengths with Wolfsohn's is given in the last two columns of Table 5, which shows that his conclusions concerning the pressure shift toward the violet are not verified. In fact, assuming the correctness of his arc-in-air measurements the residuals, Bureau of Standards minus Wolfsohn, indicate for copper the same type of pressure shift as has been found for all other spectra which have been investigated.

${ }^{20}$ Astrophys. J. 66, p. 256; 1927.

21 Phys. Rev., 28, p. 449; 1926.

${ }_{22}$ Annalen der Physik (IV), 80, p. 415; 1926. 
3. $\mathrm{Na}, \mathrm{Al}, \mathrm{Ca}, \mathrm{V}, \mathrm{Cr}, \mathrm{Mn}, \mathrm{Ni}, \mathrm{Ba}$

In Table 6 are listed some wave lengths of $\mathrm{Na}, \mathrm{Al}, \mathrm{Ca}, \mathrm{V}, \mathrm{Cr}, \mathrm{Mn}$, $\mathrm{Ni}$, and $\mathrm{Ba}$. Some of these elements, as stated above, occurred as impurities in the electrodes. $\mathrm{Ca}, \mathrm{Mn}, \mathrm{Ni}$, and $\mathrm{Ba}$ were especially investigated to furnish additional standards in the red for comparison with the solar wave lengths. The intensities and temperature classes given for each line are those assigned by King, except for $\mathrm{Na}$, and $\mathrm{Al}$, for which the estimates given by Kayser ${ }^{23}$ are quoted. The impurity lines are in all cases either the raies ultimes or persistent lines of the corresponding elements, and all appear among the Fraunhofer lines of the sun's spectrum.

\section{ACKNOWLEDGMENTS}

The work described in the foregoing pages has been in progress at the Bureau of Standards for several years. Various persons have cooperated with me in carrying it to completion, and it is a pleasure to acknowledge here my appreciation of their assistance. D. D. Laun, formerly of the bureau staff, did a large share of the observing and computations required for the work with the silveron-glass interferometers. Dr. K. Burns, of the Allegheny Observatory, shared in the observing with the platinum-on-quartz interferometers; and B. W. Scribner, jr., of the bureau staff, has assisted in the reductions of the last series of observations. Finally, to Prof. H. N. Russell, of Princeton University, I owe my thanks for placing at my disposal his unpublished results on the classification of the titanium arc and spark spectra.

Washington, December 19, 1927.

${ }^{23}$ Kayser, Tabelie der Hauptlinien. Julius Springer, Berlin, 1926. 